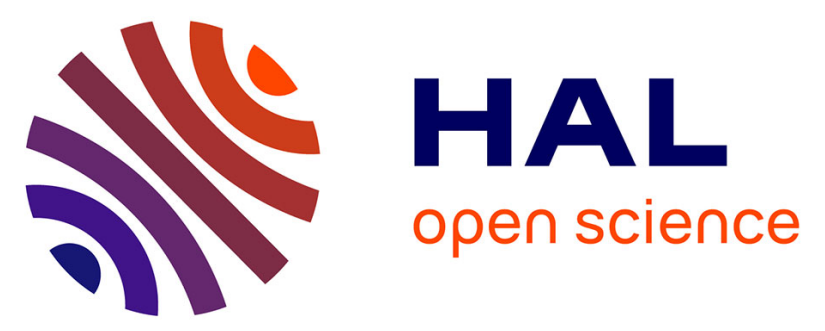

\title{
MPHT-Promoted Bromocyclization of ortho-Substituted Arylalkynes: Application to the Synthesis of 2-Substituted 3-Bromobenzofurans and -Benzo[b]thiophenes
}

\author{
Maud Jacubert, Abdellatif Tikad, Olivier Provot, Abdallah Hamze,
} Jean-Daniel Brion, Mouad Alami

\section{To cite this version:}

Maud Jacubert, Abdellatif Tikad, Olivier Provot, Abdallah Hamze, Jean-Daniel Brion, et al.. MPHTPromoted Bromocyclization of ortho-Substituted Arylalkynes: Application to the Synthesis of 2Substituted 3-Bromobenzofurans and -Benzo[b]thiophenes. European Journal of Organic Chemistry, 2010, 2010 (23), pp.4492-4500. 10.1002/ejoc.201000529 . hal-02394491

\section{HAL Id: hal-02394491 \\ https://hal.science/hal-02394491}

Submitted on 4 Dec 2019

HAL is a multi-disciplinary open access archive for the deposit and dissemination of scientific research documents, whether they are published or not. The documents may come from teaching and research institutions in France or abroad, or from public or private research centers.
L'archive ouverte pluridisciplinaire HAL, est destinée au dépôt et à la diffusion de documents scientifiques de niveau recherche, publiés ou non, émanant des établissements d'enseignement et de recherche français ou étrangers, des laboratoires publics ou privés. 


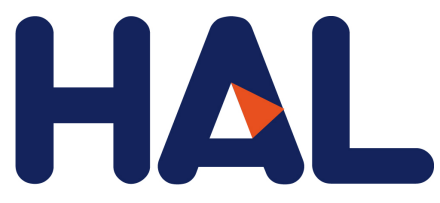

archives-ouvertes

\title{
MPHT-Promoted Bromocyclization of ortho-Substituted Arylalkynes: Application to the Synthesis of 2-Substituted 3-Bromobenzofurans and -Benzo[b]thiophenes
}

\author{
Maud Jacubert, Abdellatif Tikad, Olivier Provot, Abdallah Hamze,
} Jean-Daniel Brion, Mouad Alami

\section{To cite this version:}

Maud Jacubert, Abdellatif Tikad, Olivier Provot, Abdallah Hamze, Jean-Daniel Brion, et al.. MPHTPromoted Bromocyclization of ortho-Substituted Arylalkynes: Application to the Synthesis of 2Substituted 3-Bromobenzofurans and -Benzo[b]thiophenes. European Journal of Organic Chemistry, Wiley-VCH Verlag, 2010, pp.n/a-n/a. 10.1002/ejoc.201000529 . hal-02394491

\section{HAL Id: hal-02394491 \\ https://hal.archives-ouvertes.fr/hal-02394491}

Submitted on 4 Dec 2019

HAL is a multi-disciplinary open access archive for the deposit and dissemination of scientific research documents, whether they are published or not. The documents may come from teaching and research institutions in France or abroad, or from public or private research centers.
L'archive ouverte pluridisciplinaire HAL, est destinée au dépôt et à la diffusion de documents scientifiques de niveau recherche, publiés ou non, émanant des établissements d'enseignement et de recherche français ou étrangers, des laboratoires publics ou privés. 


\title{
MPHT Promoted Bromocyclization of Ortho Substituted Arylalkynes: Application to the Synthesis of 2-Substituted-3-Bromo-Benzofurans and -Benzo[b]thiophenes
}

\author{
Maud Jacubert, ${ }^{[\mathrm{a}]}$ Abdellatif Tikad, ${ }^{[\mathrm{a}]}$ Olivier Provot, ${ }^{[\mathrm{a}]}$ Abdallah Hamze, ${ }^{[\mathrm{a}]}$ Jean-Daniel Brion $^{[\mathrm{a}]}$ and \\ Mouâd Alami*[a]
}

Keywords: Enynes / Enediynes / Alkynes / Benzofurans / Benzothiophenes / MPHT / Cyclization.

\begin{abstract}
A convenient and general approach to the synthesis of 2-substituted-3bromobenzofurans and benzothiophenes was developed. The procedure is based on the cyclization of ortho-substituted arylalkynes in the presence of $N$-methylpyrrolidin-2-one hydrotribromide (MPHT) as a soft and easy to handle electrophilic brominating reagent. Under mild reaction conditions, MPHT promoted the bromocyclization of various enynes and diynes as well as arylalkynes to give 2-substituted3-bromobenzofurans and benzothiophenes with high to excellent yields.
\end{abstract}

Subsequent functionalization by palladium-catalyzed coupling reactions at the $\mathrm{C}-\mathrm{Br}$ bond afforded a general access to 2,3disubstituted benzofurans and benzothiophenes of biological interest.

\section{Introduction}

As part of a program focusing on the functionalization of arylalkynes, ${ }^{[1]}$ we have recently reported their reactivity with $p$ toluenesulfonic acid (PTSA) in EtOH. ${ }^{[2]}$ There are two main strands to this work, which consist of the triple bond hydration ${ }^{[3]}$ or cyclization reactions, ${ }^{[4]}$ depending on the alkyne substrates. In the hydration process promoted by PTSA, the reaction takes place rapidly in $\mathrm{EtOH}$ or water and regioselectively afforded in good yields a large variety of ketones according to Markovnikov's rules (Path A, Scheme 1).

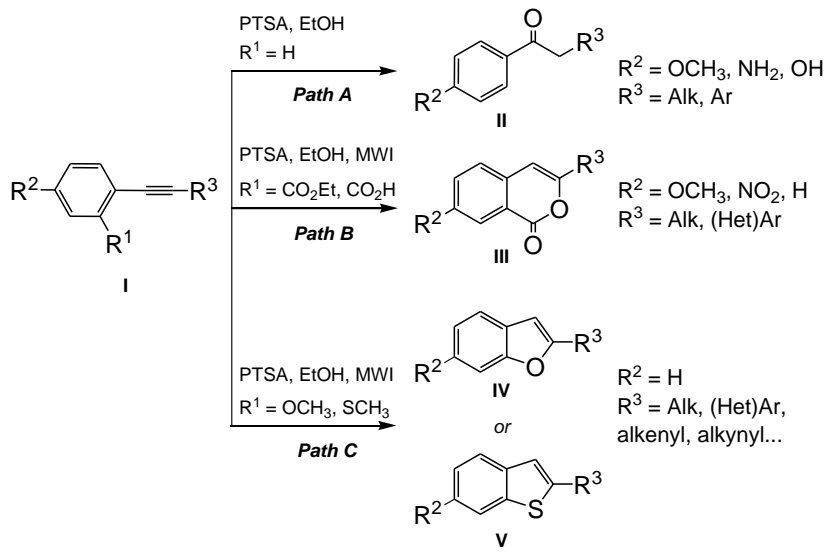

Scheme 1. Reaction of substituted arylalkynes with PTSA

[a] Univ Paris-Sud, CNRS, BioCIS UMR 8076, Laboratoire de Chimie Thérapeutique, Faculté de Pharmacie, 5 rue J.-B. Clément, ChâtenayMalabry, F-92296, France.

Tel.: +33-1-46.83.58.87; Fax: +33-1-46.83.58.28;

E-mail: mouad.alami@u-psud.fr

Supporting information for this article is available on the WWW under http://www.eurjoc.org/ or from the author

The second string of this programme involves an intra molecular cyclization reaction, promoted by PTSA, of ortho substituted diarylalkynes. With substrates bearing on the ortho position an ethoxycarbonyl or a carboxylic acid function $\left(\mathrm{CO}_{2} \mathrm{Et}, \mathrm{COOH}\right), 3$-substituted isocoumarins were obtained in good to excellent yields (path B, Scheme 1). ${ }^{[4 a]}$ When applying this environmentally metal-free procedure to ortho (1-alkynyl)anisole or thioanisole derivatives, a series of 2-substitutedbenzofurans and -benzothiophenes was prepared in good to excellent yields, respectively (path C, Scheme 1). ${ }^{[4 b]}$ In these cyclisation reactions, ${ }^{[5]}$ PTSA activated the triple bond, and a subsequent regioselective 5- or 6-endo-dig-cyclization with the ortho substituent afforded the required 2-substituted heterocycle. It should be noted that 2 -substituted- as well as 2,3-disubstituted benzofurans and benzothiophenes are attractive synthetic target molecules due to the wide spectrum of their biological activities, ${ }^{[6]}$ including antimitotic properties. ${ }^{[7]}$

In continuation of our studies concerning the desing and the preparation of anticancer agents, ${ }^{[1 \mathrm{i}, 8]}$ we were interested in developing a novel access to these class of 2,3-disubstituted heterocycles. Generally, 2-substituted 3-halo-benzofurans and benzo[$[b]$ thiophenes either are prepared by electrophilic cyclization of 2-alkynylphenol, ${ }^{[9]}$-ether, ${ }^{[6 e, f, 10]}$ and sulfide derivatives $^{[10 a, 11]}$ with a range of electrophiles $\left(\mathrm{I}_{2}, \mathrm{ICl}, \ldots\right)$. Alternative methods are based on treatment of 2-substituted benzothiophene derivatives with molecular $\mathrm{Br}_{2} .{ }^{[\mathrm{cc}]}$ Our continuing interest in the electrophilic cyclization of ortho substituted arylalkynes encouraged us to further examine the use of $\mathrm{N}$-methylpyrrolidin-2-one hydrotribromide (MPHT), developed in our $1 \mathrm{ab}^{[12]}$ as a mild electrophile, in the preparation of 2-substituted-3-bromo-benzofurans and -benzothiophene. MPHT complex is a stable solid that smoothly liberates bromine in organic solvents. It presents several advantages than molecular bromine: (i) MPHT can be stored several month at room temperature with no decrease in the free bromine titer, (ii) it is not corrosive, not necrosive, and therefore easy to weigh and to handle. Because MPHT is a mild reagent, we envisioned to used it in the bromocyclization of highly unsaturated alkynes, including 1,3-enynes and 1,3diynes to provide 3 -bromo-benzofurans and benzo $[b]$ thiophenes having an alkenyl or an alkynyl chain on the C-2 position, respectively. To our knowledge, only one example of the 
bromocyclization of an enyne substrate is described in the literature. Larock reported that a 2-thiomethylarylenyne substrate was bromocyclized using NBS for two days, whereas the bromocyclization was unsuccessfull with $\mathrm{Br}_{2}$ probably "because $\mathrm{Br}_{2}$ addition to the carbon-carbon double bond is occuring faster than cyclization". ${ }^{[11 \mathrm{~b}, \mathrm{c}]}$ We report herein an easy and versatile process for the synthesis of 2-substituted-3bromo-benzofurans and benzothiophenes from the electrophilic MPHT promoted cyclization of 1,3-enynes and 1,3-diynes as well as diarylalkynes.

Table 1. MPHT promoted the bromocyclization of ortho substituted conjugated enynes $\mathbf{1}$ and diynes $\mathbf{3}$.

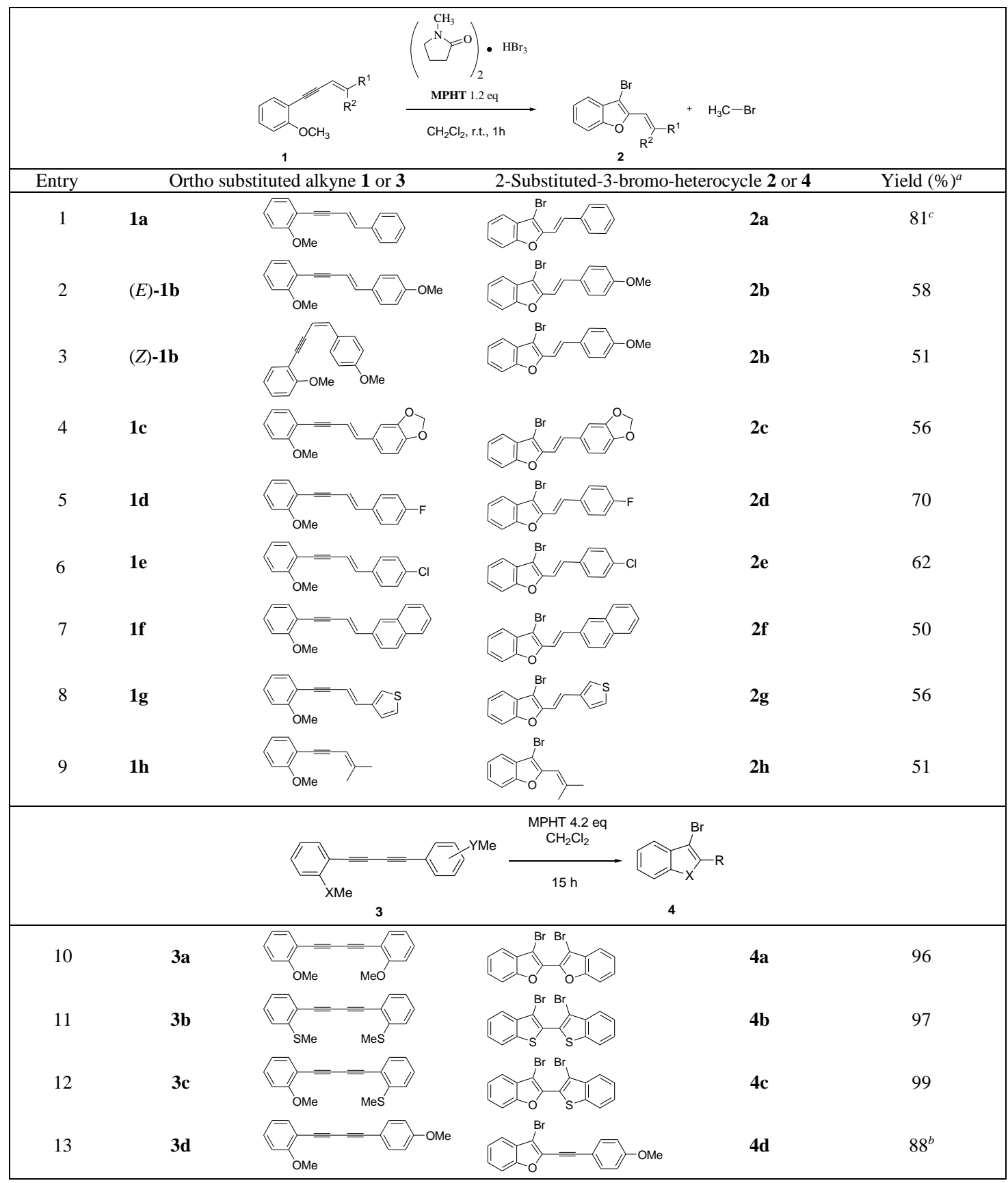

${ }^{a}$ Isolated yields. ${ }^{b} 1.5$ eq of MPHT were used for $5 \mathrm{~h} .{ }^{c}$ A complex mixture was obtained with $\mathrm{Br}_{2}$ (1.2 equiv) and $35 \%$ of $\mathbf{2 b}$ was obtained using NBS (1.2 equiv) instead of MPHT, but after $36 \mathrm{~h}$ at room temperature.

This method furnished heterocycles bearing various substituents on the $\mathrm{C}-2$ position and a bromine atom at the $\mathrm{C}-3$ position useful for further palladium-catalyzed coupling reactions.

\section{Results and Discussion}

The required 1,3-enynes $\mathbf{1}^{[13]}$ and 1,3-diynes $\mathbf{3}^{[14]}$ were prepared by Pd-catalyzed coupling reactions, according to literature procedures. Initially, 1,3-enyne substrates were evaluated in the
MPHT bromocyclization process and the results of this study are reported in Table 1. From these results, we can see that all reactions work well in $\mathrm{CH}_{2} \mathrm{Cl}_{2}$ at room temperature for $1 \mathrm{~h}$. As shown in entry 1, enyne 1a bearing no substituent on the styryl moiety reacted cleanly and rapidly with MPHT (1.2 equiv) to give the expected $(E)$-2-styryl-3-bromobenzofuran $\mathbf{2 a}$ in a good $81 \%$ yield. It is noteworthy that no product resulting from the bromination of the carbon-carbon double or triple bond of 1a was detected in the crude as judged by ${ }^{1} \mathrm{H}$ NMR. When $(E)$ - or (Z)-enynes $\mathbf{1 b}$ were employed as substrates for the 
bromocyclization, a single (E)-styrylbenzofuran $\mathbf{2 b}$ was obtained probably for thermodynamic considerations (entries 2 and 3). Enynes 1 having either electron donating (entries 2-4 and 7) or electron withdrawing substituents (entries 5 and 6) on the styryl moiety were evaluated and gave satisfactory yields in the corresponding (E)-2-substituted-3-bromobenzofurans 2 b-f. A thiophene nucleus was also tolerated on the enyne $\mathbf{1 g}$ and the expected benzofuran $\mathbf{2 g}$ was obtained in a satisfactory $56 \%$ yield (entry 8 ). Finally, the reaction was successfully achieved with enyne $\mathbf{1 h}$ bearing two methyl substituents on the double bond, thus demonstrating the general character of the method (entry 9).

Next, 1,3-diynes 3a and $\mathbf{3 b}$ were evaluated in the presence of 4.2 equiv. of MPHT (Table 1). We were pleased to observe the quantitative formation of bis-3-bromobenzofuran $\mathbf{4 a}$ and bis-3bromobenzothiophene $\mathbf{4 b}$ which have been used as starting materials for the synthesis of benzannelated phospholes, ${ }^{15}$ dibenzothienopyrroles, ${ }^{[16]}$ dithienosiloles ${ }^{[17]}$ and functionalized phenanthrenes ${ }^{[18]}$ of physical interest. Example depicted in entry 12 showed that unsymmetrical diyne $\mathbf{3 c}$ reacted cleanly with MPHT to afford the expected heterocycle $\mathbf{4 c}$, again with excellent yield. Finally, the selectivity observed in the presence of conjugated diyne 3d must be especially underlined as the bromocyclization reaction efficiently occurred to provide $\mathbf{4 d}$, with no addition of bromine to the second carbon-carbon triple bond (entry 13).

Table 2. MPHT promoted the bromocyclization of ortho-substituted diarylalkynes 7
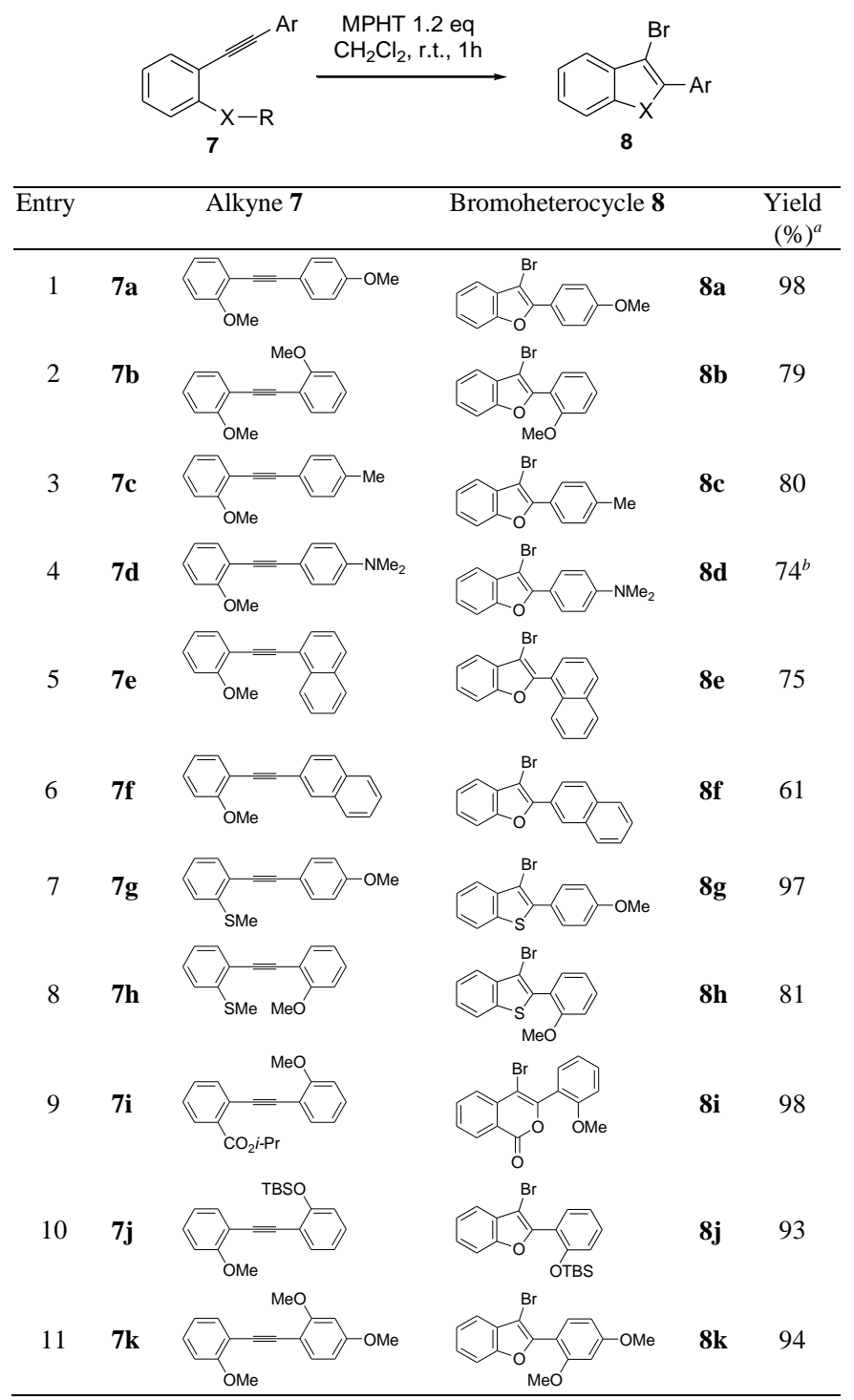

${ }^{a}$ Isolated yields. ${ }^{b} 3 \mathrm{~h}$ were required.

Having demonstrated the efficiency of MPHT to promote the bromocyclization of conjugated enynes $\mathbf{1}$ and diynes $\mathbf{3}$, we sought to extend the cyclization reaction with highly conjugated (E)-enediyne 5. ${ }^{[19]}$ Thus, after stirring with MPHT (2.4 equiv) at room temperature for $3 \mathrm{~h}$, we were pleased to observe the formation of 1,2-ethenyl bis-benzofuran $\mathbf{6}$ which was isolated in a moderate $21 \%$ yield, despite the fact that the reaction conditions have not been optimized (Scheme 2). A further cyclization of $\mathbf{6}$ (or its benzothiophene analogue) would provide a set of fused thiepins ${ }^{[20]}$ as well as 1-sila, 1-germa, 1selenacyclohepta-2,4,6-trienes ${ }^{[21]}$ of biological interest. 


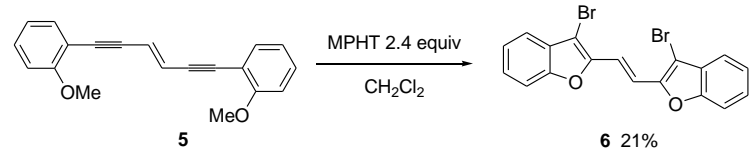

Scheme 2. Bromocyclization of (E)-enediyne 5 with MPHT.

Having succeeded in developing an efficient bromocyclization of conjugated enyne, diyne and enediyne substrates, we next examined the reaction with a range of ortho substituted diarylalkynes 7, which were prepared by Pd-catalyzed coupling reactions according to literature procedures. ${ }^{[22]}$ The results of this study are summarized in Table 2. Under the above reaction conditions, MPHT promoted annulations of 2-methoxy diarylalkynes 7a-f and provided good yields of the corresponding 2-aryl-3-bromobenzofurans 8a-f (entries 1-6). Similarly, 2-thioanisole derivative $\mathbf{7 g}$ undergoes a rapid cyclization to give 2-aryl-3-bromobenzothiophene $\mathbf{8 g}$ in a nearly quantitative yield (entry 7, 97\%). To determine the relative reactivity of various substituents toward the bromocyclization, the MPHT protocol was applied to unsymmetrically 2,2'disubstituted diarylalkynes $\mathbf{7 h}-\mathbf{k}$. Alkyne $\mathbf{7 h}$ with an ortho methoxy- and ortho' thiomethyl- substituents undergoes bromocyclization at the sulphur atom to give selectively benzothiophene $\mathbf{8 h}$ in a good yield (entry 8). A similar selectivity was observed with diarylalkyne $7 \mathbf{i}$ having an ortho ester function (entry 9). In this case, the 6-endo-dig cyclization proceeded selectively to provide exclusively isocoumarin $\mathbf{8 i}$ in an excellent $98 \%$ yield. In example depicted in entry 10 , we have observed a total hierarchy of a OMe substituent versus a OTBDMS group toward the bromocyclization and the reaction gave 3-bromobenzofuran $\mathbf{8 j}$ having a OTBDMS substituent on the C-2'. Surprisingly, starting from $o, o^{\prime}, p$-trimethoxyalkyne $7 \mathbf{k}$, a single cyclization product $\mathbf{8 k}$ was formed showing a significant difference in reactivity between the two ortho methoxy substituents of $\mathbf{7 k}$ (entry $11,94 \%$ ).

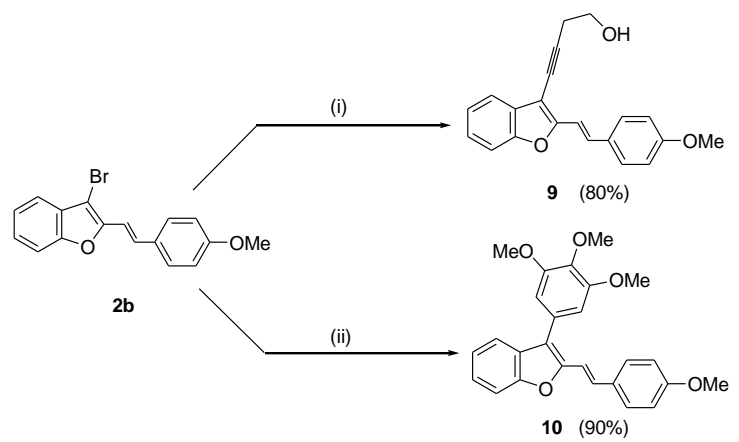

Scheme 3. Cross-coupling reactions of $\mathbf{2 b}$ under palladium catalysis. Reagents and conditions: (i). $\mathrm{CuI}(10 \mathrm{~mol} \%), \mathrm{PdCl}_{2}\left(\mathrm{PPh}_{3}\right)_{2}(5 \mathrm{~mol} \%)$, but-1-yn-4-ol (1.6 equiv), $\quad \mathrm{Et}_{3} \mathrm{~N}, \quad 50{ }^{\circ} \mathrm{C}, \quad 12$ h. $\quad$ (ii) 3,4,5trimethoxyphenylboronic acid (1.2 equiv), $\mathrm{Pd}\left(\mathrm{PPh}_{3}\right)_{4}(5 \mathrm{~mol} \%), \mathrm{K}_{2} \mathrm{CO}_{3}$ (2 equiv), toluene- $\mathrm{H}_{2} \mathrm{O}(2 / 1), 100{ }^{\circ} \mathrm{C}$.

To increase the synthetic utility of these 2-substituted-3bromobenzofurans, one the cyclization product, (E)-2-(4methoxystyryl)-3-bromobenzofuran $\mathbf{2 b}$ was converted to 2alkenyl-3-alkynylbenzofuran 9 and 2-alkenyl-3-arylbenzofuran 10 by Sonogashira and Suzuki cross-coupling reactions with but-1-yn-4-ol and 3,4,5-trimethoxyphenylboronic acid, respectively in good yields (Scheme 3). One can note that compound $\mathbf{1 0}$ may be regarded as a conformationally restricted analogue of vinylogous combretastatin A-4 of biological interest. ${ }^{[23]}$

\section{Conclusions}

In summary, we have showed that MPHT in $\mathrm{CH}_{2} \mathrm{Cl}_{2}$ is a soft and compatible reagent to promote the bromocyclization of ortho substituted alkynes at room temperature. Under these mild conditions, it was demonstrated that various conjugated enyne, diyne, enediyne and arylalkyne substrates were successfully cyclized in good to excellent yields to provide structurally interesting 2-substituted-3-bromobenzofuran and benzothiophene derivatives. By further chemical manipulations, our synthetic approach should allow rapid access to a wide range of 2,3-disubstituted benzofurans and benzothiophenes amenable for biological evaluations.

\section{Experimental details}

\section{General comments}

All glasswares were oven-dried at $140{ }^{\circ} \mathrm{C}$ and all reactions were conducted under a nitrogen atmosphere. Solvents were dried by standard methods and distilled before use. Piperidine was dried and distilled from potassium hydroxide prior to use. $\mathrm{Pd}\left(\mathrm{PPh}_{3}\right)_{4}$ was prepared following literature procedure. ${ }^{[2]}$ The compounds were all identified by usual physical methods, i.e. ${ }^{1} \mathrm{H}-\mathrm{NMR}$, ${ }^{13} \mathrm{C}-\mathrm{NMR}$, IR, MS and elemental analysis. ${ }^{1} \mathrm{H}$ and ${ }^{13} \mathrm{C}$ NMR spectra were measured in $\mathrm{CDCl}_{3}$ with a Bruker Avance 300 or Bruker ARX 400. ${ }^{1} \mathrm{H}$ chemical shifts are reported in ppm from the peak of residual chloroform (7.27 ppm) and coupling constants $J$ were measured in $\mathrm{Hz}$. The following abreviation are use: $\mathrm{m}$ (multiplet), s (singlet), d (doublet), $\mathrm{t}$ (triplet) dd (doublet of doublet), bs (broad singlet). ${ }^{13} \mathrm{C}$ chemical shifts are reported in $\mathrm{ppm}$ from the central peak of deuteriochloroform (77.14). IR spectra were measured on a Bruker Vector 22 spectrophotometer (neat, $\mathrm{cm}^{-1}$ ). Elemental analyses (for all new cyclized products) were performed with a Perkin-Elmer 240 analyser. Mass spectra were obtained using a Bruker Esquire electrospray ionization apparatus. Analytical TLC was performed on Merck precoated silica gel $60 \mathrm{~F}$ plates. Merck silica gel 60 (230-400 mesh) was used for column chromatography. Enynes 1a-c, 1e-g, enediynes 3a-b and diarylalkynes 5a-k were prepared according to literature procedures.

\section{Experimental procedures}

(E)-1-Fluoro-4-(4-(2-methoxyphenyl)but-1-en-3-ynyl)-benzene (1d) $(214 \mathrm{mg} ; 85 \%)$. To a mixture of $(E)-1$-(4-chlorobut-3en-1-ynyl)-2-methoxybenzenechloroenyne $(192 \mathrm{mg}, 1 \mathrm{mmol})$ in toluene $(4 \mathrm{~mL})$ and EtOH $(2 \mathrm{~mL})$, were successively added 4fluorophenylboronic acid (168 mg, $1.2 \mathrm{mmol}), \mathrm{K}_{2} \mathrm{CO}_{3}(276 \mathrm{mg}$, $2 \mathrm{mmol})$ and $\mathrm{Pd}\left(\mathrm{PPh}_{3}\right)_{4}(58 \mathrm{mg}, 0.05 \mathrm{mmol})$. The reaction was heated at $100{ }^{\circ} \mathrm{C}$, under vigorous stirring and monitored by TLC until complete disappearance of starting material. The solvent was evaporated in vacuo and $10 \mathrm{~mL}$ of water were added. After extraction with $\mathrm{CH}_{2} \mathrm{Cl}_{2}(3 \times 10 \mathrm{~mL})$, the combined organic layers were dried over $\mathrm{MgSO}_{4}$ and the solvent was removed under reduced pressure. The crude material was purified by column chromatography to afford the expected enyne 1d. ${ }^{1} \mathrm{H}$ $\mathrm{NMR}\left(\mathrm{CDCl}_{3}, 300 \mathrm{MHz}\right): \delta 3.91\left(\mathrm{~s}, 3 \mathrm{H}, \mathrm{OCH}_{3}\right), 6.37(\mathrm{~d}, J=$ $\left.16.2 \mathrm{~Hz}, 1 \mathrm{H}, \mathrm{H}_{\text {vinyl }}\right), 6.88-6.96\left(\mathrm{~m}, 2 \mathrm{H}, \mathrm{H}_{\text {arom }}\right), 7.00-7.06(\mathrm{~m}, 3 \mathrm{H}$, $\left.\mathrm{H}_{\text {arom }+ \text { vinyl }}\right), 7.30\left(\mathrm{dt}, J=7.9 \mathrm{~Hz}, J=1.7 \mathrm{~Hz}, 1 \mathrm{H}, \mathrm{H}_{\text {arom }}\right), 7.39$ (dd, $\left.J=8.8 \mathrm{~Hz}, J=5.4 \mathrm{~Hz}, 2 \mathrm{H}, \mathrm{H}_{\text {arom }}\right), 7.45(\mathrm{dd}, J=7.5, J=1.7$ $\mathrm{Hz}, 1 \mathrm{H}) .{ }^{13} \mathrm{C} \mathrm{NMR}\left(\mathrm{CDCl}_{3}, 75 \mathrm{MHz}\right): \delta 55.9\left(\mathrm{CH}_{3}\right), 88.2(\mathrm{Cq})$, $92.8(\mathrm{Cq}), 108.4(\mathrm{CH}), 110.7(\mathrm{CH}), 112.6(\mathrm{Cq}), 115.8\left(2 \mathrm{CH}, J_{\mathrm{CF}}\right.$ 
$=21.8 \mathrm{~Hz}), 120.6(\mathrm{CH}), 128.0\left(2 \mathrm{CH}, J_{\mathrm{CF}}=8.1 \mathrm{~Hz}\right), 129.9(\mathrm{CH})$, $132.8\left(\mathrm{Cq}, J_{\mathrm{CF}}=3.1 \mathrm{~Hz}\right), 133.6(\mathrm{CH}), 139.7(\mathrm{CH}), 160.0(\mathrm{Cq})$, $162.9\left(\mathrm{Cq}, J_{\mathrm{CF}}=248.6 \mathrm{~Hz}\right)$. IR $\left(v^{-1} \mathrm{~cm}^{-1}\right): 1597,1490,1434,1228$, $1158,1024,954,813,750$. MS (APCI+) $m / z, 253.0(\mathrm{M}+\mathrm{H})^{+}$.

1-Methoxy-2-(4-methylpent-3-en-1-ynyl)benzene (1h) (312 $\mathrm{mg} ; 70 \%$ ).

To a solution of $\mathrm{PdCl}_{2}\left(\mathrm{PPh}_{3}\right)_{2}(104 \mathrm{mg}, 0.15 \mathrm{mmol}), \mathrm{CuI}(56.4$ $\mathrm{mg}, 0.3 \mathrm{mmol})$ and bromo-1-methylpropene $(400 \mathrm{mg}, 3 \mathrm{mmol})$ in THF $(10 \mathrm{~mL})$ and piperidine $(1.2 \mathrm{~mL} ; 11.9 \mathrm{mmol})$ was slowly added 2-methoxyphenylacetylene (461 $\mu \mathrm{L} ; 2.4 \mathrm{mmol})$. The stirred reaction was refluxed for $20 \mathrm{~h}$ and treated with a saturated $\mathrm{NH}_{4} \mathrm{Cl}$ solution $(10 \mathrm{~mL})$. The aqueous layer was extracted with EtOAc $(3 \times 10 \mathrm{~mL})$ and the combined organic layers were dried over $\mathrm{MgSO}_{4}$ and concentrated under vacuum. Purification by flash chromatography afforded the expected enyne 1h. $\mathrm{R}_{\mathrm{f}}=0.41$ (Cyclohexane/ $\left.\mathrm{CH}_{2} \mathrm{Cl}_{2}: 8 / 2\right)$. NMR ${ }^{1} \mathrm{H}$ $\left(\mathrm{CDCl}_{3}, 300 \mathrm{MHz}\right): \delta 1.87\left(\mathrm{~s}, 3 \mathrm{H}, \mathrm{CH}_{3}\right), 2.01\left(\mathrm{~s}, 3 \mathrm{H}, \mathrm{CH}_{3}\right), 3.88$ (s, 3H, $\left.\mathrm{OCH}_{3}\right), 5.54$ (bs, $\left.1 \mathrm{H}, \mathrm{H}_{\text {vinyl }}\right), 6.87(\mathrm{~d}, J=8.2 \mathrm{~Hz}, 1 \mathrm{H}$, $\left.\mathrm{H}_{\text {arom }}\right), 6.90\left(\mathrm{t}, J=7.5 \mathrm{~Hz}, 1 \mathrm{H}, \mathrm{H}_{\text {arom }}\right), 7.25(\mathrm{t}, J=7.9 \mathrm{~Hz}, 1 \mathrm{H}$, $\left.\mathrm{H}_{\text {arom }}\right), 7,40\left(\mathrm{~d}, J=7,5 \mathrm{~Hz}, 1 \mathrm{H}, \mathrm{H}_{\text {arom }}\right) . \mathrm{NMR}{ }^{13} \mathrm{C}\left(\mathrm{CDCl}_{3}, 75\right.$ $\mathrm{MHz}): \delta 21.2\left(\mathrm{CH}_{3}\right) ; 25.0\left(\mathrm{CH}_{3}\right), 55.9\left(\mathrm{OCH}_{3}\right), 87.8(\mathrm{C}), 92.1$ (C), $105.7(\mathrm{CH}), 110.7(\mathrm{CH}), 113.4(\mathrm{C}), 120.5(\mathrm{CH}), 129.2$ $(\mathrm{CH}), 133.2(\mathrm{CH}), 148.8(\mathrm{C}), 159.7(\mathrm{C}) . \mathrm{IR}\left(\mathrm{v} \mathrm{cm}^{-1}\right): 2908$, $1593,1434,1268,1240,1119,1047,749$. MS (APCI+) $\mathrm{m} / \mathrm{z}$ $373.0\left([2 \mathrm{M}+\mathrm{H}]^{+}\right), 187.0\left([\mathrm{M}+\mathrm{H}]^{+}\right)$.

Preparation of unsymmetrical diynes (3c-d) To a solution of $\mathrm{CuI}(0.1 \mathrm{mmol})$ in pyrrolidine $(10 \mathrm{~mL})$ was added successively at $0{ }^{\circ} \mathrm{C}, 1 \mathrm{mmol}$ of iodoarylalkyne and $2 \mathrm{mmol}$ of terminal arylalkyne. The stirred reaction was kept at room temperature for a night and treated with a saturated $\mathrm{NH}_{4} \mathrm{Cl}$ solution $(15 \mathrm{~mL})$. The aqueous layer was extracted with EtOAc $(3 \times 10 \mathrm{~mL})$ and the combined organic layers were dried over $\mathrm{MgSO}_{4}$ and concentrated under vacuum. Purification by flash chromatography afforded the expected diynes

\section{(2-(4-(2-Methoxyphenyl)buta-1,3-diynyl)phenyl)(methyl)} sulfane (3c) ${ }^{[25]}(152 \mathrm{mg} ; 70 \%)$. Prepared from CuI (14.8 mg, $0.08 \mathrm{mmol}), 1$-(2-iodoethynyl)-2-methoxybenzene (200 mg, $0.78 \mathrm{mmol}$ ) and 2-thiomethylphenylacetylene. $\mathrm{Mp}=82-83{ }^{\circ} \mathrm{C}$. $\mathrm{R}_{\mathrm{f}}=0.22$ (Cyclohexane/EtOAc: 94/6). NMR ${ }^{1} \mathrm{H}\left(\mathrm{CDCl}_{3}, 400\right.$ $\mathrm{MHz}): \delta 2.51\left(\mathrm{~s}, 3 \mathrm{H}, \mathrm{SCH}_{3}\right), 3.91\left(\mathrm{~s}, 3 \mathrm{H}, \mathrm{OCH}_{3}\right), 6.89(\mathrm{~d}, J=$ $\left.8.7 \mathrm{~Hz}, 1 \mathrm{H}, \mathrm{H}_{\text {arom }}\right), 6.92\left(\mathrm{t}, J=7.5 \mathrm{~Hz}, 1 \mathrm{H}, \mathrm{H}_{\text {arom }}\right), 7.09(\mathrm{t}, J=$ $\left.7.5 \mathrm{~Hz}, 1 \mathrm{H}, \mathrm{H}_{\text {arom }}\right), 7.17$ (d, J=7.9 Hz, 1H, $\left.\mathrm{H}_{\text {arom }}\right), 7.29-7.36$ (m, $\left.2 \mathrm{H}, \mathrm{H}_{\text {arom }}\right), 7.48-7.51$ (m, $\left.2 \mathrm{H}, \mathrm{H}_{\text {arom }}\right)$. NMR ${ }^{13} \mathrm{C}\left(\mathrm{CDCl}_{3}, 100\right.$ $\mathrm{MHz}): \delta 15.4\left(\mathrm{SCH}_{3}\right), 56.0\left(\mathrm{OCH}_{3}\right), 77.7,79.5,80.1,80.4$, $110.8,111.2,120.5,120.7,124.5,124.6,129.5,130.9$, 133.7, 134.6, 143.2, 161.5. IR $\left(v^{-1}\right): 2938,1594,1492,1464,1433$, $1248,1022,733,703$. MS (APCI+) $m / z 279.0\left([\mathrm{M}+\mathrm{H}]^{+}\right)$.

\section{1-(2-Methoxyphenyl)-4-(4-methoxyphenyl)buta-1,3-diyne}

(3d) (131 mg; 64\%). Prepared from CuI (40.6 mg, $0.21 \mathrm{mmol})$, 1-(2-iodoethynyl)-2-methoxybenzene (550 $\mathrm{mg}, 0.78 \mathrm{mmol}$ ) and 4-methoxyphenylacetylene. $\mathrm{Mp}=109-110{ }^{\circ} \mathrm{C} . \quad \mathrm{R}_{\mathrm{f}}=0.24$ (Cyclohexane/Acetone: 95/5). $\mathrm{NMR}{ }^{1} \mathrm{H}\left(\mathrm{CDCl}_{3}, 300 \mathrm{MHz}\right)$ : $\delta 3.82\left(\mathrm{~s}, 3 \mathrm{H}, \mathrm{OCH}_{3}\right), 3.90\left(\mathrm{~s}, 3 \mathrm{H}, \mathrm{OCH}_{3}\right), 6.84-6.94(\mathrm{~m}, 4 \mathrm{H}$, $\mathrm{H}_{\text {arom }}$ ), 7.32 (t, J=7.6 Hz, 1H, $\left.\mathrm{H}_{\text {arom }}\right), 7.45-7.48$ (m, 3H, $\left.\mathrm{H}_{\text {arom }}\right)$. $\mathrm{NMR}{ }^{13} \mathrm{C}\left(\mathrm{CDCl}_{3}, 75 \mathrm{MHz}\right): \delta 55.4\left(\mathrm{OCH}_{3}\right), 55.9\left(\mathrm{OCH}_{3}\right), 73.2$, 77.7, 78.0, 82.5, 110.8, 111.3, 114.0, 114.2, 120.6, 130.6, 134.1, 134.4, 160.4, 161.5. IR ( $\left.\mathrm{v} \mathrm{cm}^{-1}\right): 2936,2837,1601,1508,1489$, 1247, 1171, 1024, 750._MS (APCI+) $m / z, 263.0\left([\mathrm{M}+\mathrm{H}]^{+}\right)$.

General procedure for the electrophilic cyclization with MPHT.
To a solution of $\mathbf{1}, \mathbf{3}$ or $\mathbf{5}$ in $\mathrm{CH}_{2} \mathrm{Cl}_{2}$ (1 mmol) was added MPHT (for quantity, see text) and the resulting solution was stirred at room temperature until disappearance of starting material (as judged by TLC). The reaction mixture was next treated with a saturated $\mathrm{Na}_{2} \mathrm{~S}_{2} \mathrm{O}_{3}$ solution. The organic layer was washed with $\mathrm{HCl} 10 \%$ (3 x $10 \mathrm{~mL}$ ) and dried over $\mathrm{MgSO}_{4}$. Removal of the solvent yielded a crude product, which was purified by silica gel flash chromatography to afford 2, 4, 6 or 8 .

(E)-3-Bromo-2-styrylbenzofuran (2a) (242 mg; 81\%). $\mathrm{R}_{\mathrm{f}}=$ 0.57 (Cyclohexane/ $\mathrm{CH}_{2} \mathrm{Cl}_{2}$ : 95/5). $\mathrm{NMR}{ }^{1} \mathrm{H}\left(\mathrm{CDCl}_{3}, 400 \mathrm{MHz}\right)$ : $\delta 7.14\left(\mathrm{~d}, J=16,2 \mathrm{~Hz}, 1 \mathrm{H}, \mathrm{H}_{\text {vinyl }}\right), 7.28-7.43\left(\mathrm{~m}, 5 \mathrm{H}, \mathrm{H}_{\text {arom }}\right)$, $7.41\left(\mathrm{~d}, J=16.2 \mathrm{~Hz}, 1 \mathrm{H}, \mathrm{H}_{\text {vinyl }}\right), 7.47\left(\mathrm{~d}, J=8.1 \mathrm{~Hz}, 1 \mathrm{H}, \mathrm{H}_{\text {arom }}\right)$, $7.50\left(\mathrm{~d}, J=7.6 \mathrm{~Hz}, 1 \mathrm{H}, \mathrm{H}_{\text {arom }}\right), 7.59$ (d, $\left.J=7.6 \mathrm{~Hz}, 2 \mathrm{H}, \mathrm{H}_{\text {arom }}\right)$. $\mathrm{RMN}{ }^{13} \mathrm{C}\left(\mathrm{CDCl}_{3}, 100 \mathrm{MHz}\right): \delta 97.0,111.3,113.8,119.7$, 123.6, 126.0, 127.1, 128.7, 128.9, 129.0, 132.0, 136.5, 151.5, 153.7. IR $\left(v^{-1} \mathrm{~cm}^{-1}\right): 3058,2924,1446,1013,1001,954,741$, 689. MS (APCI+) $\mathrm{m} / \mathrm{z} 220.0\left([\mathrm{M}-\mathrm{Br}+\mathrm{H}]^{+}\right)$. Anal. $\mathrm{C}_{16} \mathrm{H}_{11} \mathrm{BrO}$ (299.16): found C 64.09, H 3.56 requires C 64.24, H 3.71 .

(E)-2-(4-Methoxystyryl)-3-bromobenzofuran (2b) (191 mg; $58 \%)$. Beige solid. Mp 85-86 ${ }^{\circ} \mathrm{C} .{ }^{1} \mathrm{H}$ NMR $\left(\mathrm{CDCl}_{3}, 300 \mathrm{MHz}\right)$ : $\delta 3.85\left(\mathrm{~s}, 3 \mathrm{H}, \mathrm{CH}_{3}\right), 6.93\left(\mathrm{~d}, 2 \mathrm{H}, J=8.6 \mathrm{~Hz}, \mathrm{H}_{\text {arom }}\right), 6.99(\mathrm{~d}, 1 \mathrm{H}$, $\left.J=16.2 \mathrm{~Hz}, \mathrm{H}_{\text {vinyl }}\right), 7.28-7.39\left(\mathrm{~m}, 3 \mathrm{H}, \mathrm{H}_{\text {vinyl }+ \text { arom }}\right), 7.44-7.50(\mathrm{~m}$, $\left.2 \mathrm{H}, \mathrm{H}_{\text {arom }}\right), 7.53\left(\mathrm{~d}, 2 \mathrm{H}, J=8.6 \mathrm{~Hz}, \mathrm{H}_{\text {arom }}\right) .{ }^{13} \mathrm{C} \mathrm{NMR}\left(\mathrm{CDCl}_{3}\right.$, $75 \mathrm{MHz}): \delta 55.5\left(\mathrm{CH}_{3}\right), 96.0(\mathrm{C}), 111.1(\mathrm{CH}), 111.6(\mathrm{CH})$, $114.4(2 \mathrm{CH}), 119.5(\mathrm{CH}), 123.5(\mathrm{CH}), 125.6(\mathrm{CH}), 128.5(2 \mathrm{CH})$, $129.1(\mathrm{C}), 129.2(\mathrm{C}), 131.6(\mathrm{CH}), 151.8(\mathrm{Cq}), 153.6(\mathrm{C}), 160.2$ (C). IR $\left(\mathrm{v} \mathrm{cm}^{-1}\right): 821,953,1014,1173,1243,1450,1505,1599$, 2926. MS (APCI+) m/z $329.0(\mathrm{M}+\mathrm{H})^{+}\left({ }^{79} \mathrm{Br}\right), 331.0(\mathrm{M}+\mathrm{H})^{+}$ $\left({ }^{81} \mathrm{Br}\right), 250.0[(\mathrm{M}-\mathrm{Br})+\mathrm{H}]^{+} . \mathrm{C}_{17} \mathrm{H}_{13} \mathrm{BrO}_{2}$ (329.19): found $\mathrm{C}$ 61.91, H 3.84 requires C 62.03, H 3.98.

(E)-5-(2-(3-bromobenzofuran-2-yl)vinyl)benzo-[1,3]-dioxole (2c) $(192 \mathrm{mg} ; 56 \%)$. Yellow solid. Mp $122-123{ }^{\circ} \mathrm{C} .{ }^{1} \mathrm{H}$ NMR $\left(\mathrm{CDCl}_{3}, 300 \mathrm{MHz}\right): 6.00\left(\mathrm{~s}, 2 \mathrm{H}, \mathrm{OCH}_{2} \mathrm{O}\right), 6.83(\mathrm{~d}, 1 \mathrm{H}, J=8.0$ $\left.\mathrm{Hz}, \mathrm{H}_{\text {arom }}\right), 6.94$ (d, $\left.1 \mathrm{H}, J=16.1 \mathrm{~Hz}, \mathrm{H}_{\text {vinyl }}\right), 7.03$ (dd, $1 \mathrm{H}, J=$ 8.0, $\left.1.3 \mathrm{~Hz}, \mathrm{H}_{\text {arom }}\right), 7.12$ (d, $\left.1 \mathrm{H}, J=1.6 \mathrm{~Hz}, \mathrm{H}_{\text {arom }}\right), 7.27-7.35$ (m, $\left.3 \mathrm{H}, \mathrm{H}_{\text {vinyl+arom }}\right), 7.43-7.49$ (m, $\left.2 \mathrm{H}, \mathrm{H}_{\text {arom }}\right) .{ }^{13} \mathrm{C} \mathrm{NMR}\left(\mathrm{CDCl}_{3}, 75\right.$ $\mathrm{MHz}): \delta 96.4(\mathrm{C}), 101.5(\mathrm{C}), 105.9(\mathrm{CH}), 108.7(\mathrm{CH}), 111.2$ $(\mathrm{CH}), 112.0(\mathrm{CH}), 119.5(\mathrm{CH}), 122.6(\mathrm{CH}), 123.6(\mathrm{CH}), 125.8$ $(\mathrm{CH}), 129.0(\mathrm{C}), 131.0(\mathrm{C}), 131.6(\mathrm{CH}), 148.5(\mathrm{C}), 151.6(\mathrm{C})$, 153.6 (C). IR $\left(v^{-1}\right): 746,931,1037,1199,1250,1447,1487$, 2360. MS (APCI+) $\mathrm{m} / \mathrm{z} 343.0(\mathrm{M}+\mathrm{H})^{+}\left({ }^{79} \mathrm{Br}\right), 345.0(\mathrm{M}+\mathrm{H})^{+}$ $\left({ }^{81} \mathrm{Br}\right)$. Anal. $\mathrm{C}_{17} \mathrm{H}_{11} \mathrm{BrO}_{3}$ (343.17): found $\mathrm{C} 59.41, \mathrm{H} 3.16$ requires C 59.50, H 3.23.

(E)-2-(4-Fluorostyryl)-3-bromobenzofuran (2d) (222 mg; $70 \%)$. Yellow solid. Mp 83-84 ${ }^{\circ} \mathrm{C} .{ }^{1} \mathrm{H}$ NMR $\left(\mathrm{CDCl}_{3}, 300 \mathrm{MHz}\right)$ : $\delta 6.94\left(\mathrm{~d}, 1 \mathrm{H}, J=16.0 \mathrm{~Hz}, \mathrm{H}_{\text {vinyl }}\right), 7.01(\mathrm{~d}, 2 \mathrm{H}, J=8.6 \mathrm{~Hz}$, $\left.\mathrm{H}_{\text {arom }}\right)$, 7.17-7.29 (m, 3H, $\left.\mathrm{H}_{\text {arom }}\right), 7.36-7.48\left(\mathrm{~m}, 4 \mathrm{H}, \mathrm{H}_{\text {arom }}\right) .{ }^{13} \mathrm{C}$ NMR $\left(\mathrm{CDCl}_{3}, 75 \mathrm{MHz}\right): \delta 97.0(\mathrm{C}), 111.2(\mathrm{CH}), 113.6\left(\mathrm{CH}, J_{\mathrm{CF}}\right.$ $=1.5 \mathrm{~Hz}), 116.0\left(2 \mathrm{CH}, J_{\mathrm{CF}}=21.9 \mathrm{~Hz}\right), 119.7(\mathrm{CH}), 123.7(\mathrm{CH})$, $126.0(\mathrm{CH}), 128.7\left(2 \mathrm{CH}, J_{\mathrm{CF}}=8.1 \mathrm{~Hz}\right), 129.0(\mathrm{C}), 130.7(\mathrm{CH})$, $132.7\left(\mathrm{Cq}, J_{\mathrm{CF}}=3.0 \mathrm{~Hz}\right), 151.3(\mathrm{C}), 153.7(\mathrm{C}), 163.0\left(\mathrm{Cq}, J_{\mathrm{CF}}=\right.$ $249.1 \mathrm{~Hz})$. IR $\left(v^{-1} \mathrm{~cm}^{-1}\right): 740,811,847,947,1002,1151,1227$, $1447,1501,1592$. MS (APCI+) $m / z 317.0(\mathrm{M}+\mathrm{H})^{+}\left({ }^{79} \mathrm{Br}\right), 319.0$ $(\mathrm{M}+\mathrm{H})^{+}\left({ }^{81} \mathrm{Br}\right)$. Anal. $\mathrm{C}_{16} \mathrm{H}_{10} \mathrm{BrFO}$ (317.15): found $\mathrm{C} 60.47, \mathrm{H}$ 3.01 requires C 60.59, H 3.18.

(E)-2-(4-Chlorostyryl)-3-bromobenzofuran (2e) (207 mg; $62 \%)$. Yellow solid. Mp 114-115 ${ }^{\circ} \mathrm{C} .{ }^{1} \mathrm{H}$ NMR $\left(\mathrm{CDCl}_{3}, 300\right.$ MHz): 7.09 (d, $\left.1 \mathrm{H}, J=16.2 \mathrm{~Hz}, \mathrm{H}_{\text {vinyl }}\right), 7.29-7.38(\mathrm{~m}, 5 \mathrm{H}$, $\left.\mathrm{H}_{\text {vinyl+arom }}\right), 7.45-7.52(\mathrm{~m}, 4 \mathrm{H}, \mathrm{H}$ arom $) .{ }^{13} \mathrm{C} \mathrm{NMR}\left(\mathrm{CDCl}_{3}, 75\right.$ MHz): $\delta 97.5(\mathrm{C}), 111.3(\mathrm{CH}), 114.2(\mathrm{CH}), 119.8(\mathrm{CH}), 123.7$ 
$(\mathrm{CH}), 126.2(\mathrm{CH}), 128.2(2 \mathrm{CH}), 128.9(\mathrm{C}), 129.2(2 \mathrm{CH}), 130.5$ $(\mathrm{CH}), 134.3(\mathrm{C}), 135.0(\mathrm{C}), 151.2(\mathrm{C}), 153.8(\mathrm{C}) . \mathrm{IR}\left(\mathrm{v} \mathrm{cm}^{-1}\right)$ : 812, 954, 1013, 1091, 1192, 1252, 1448, 1489, 1592. MS (APCI+) $\mathrm{m} / \mathrm{z} 333.0(\mathrm{M}+\mathrm{H})^{+}\left({ }^{79} \mathrm{Br}\right), 335.0(\mathrm{M}+\mathrm{H})^{+}\left({ }^{81} \mathrm{Br}\right)$. Anal. $\mathrm{C}_{16} \mathrm{H}_{10} \mathrm{BrClO}$ (333.61): found $\mathrm{C} 333.48, \mathrm{H} 2.94$ requires $\mathrm{C}$ 57.60, H 3.02.

(E)-3-Bromo-2-(2-(naphthalen-2-yl)vinyl)benzofuran (2f) (175 mg; 50\%). Yellow solid. Mp 141-142 ${ }^{\circ} \mathrm{C} .{ }^{1} \mathrm{H} \mathrm{NMR}\left(\mathrm{CDCl}_{3}\right.$, $300 \mathrm{MHz})$ : 7.22-7.39 (m, 3H, $\left.\mathrm{H}_{\text {vinyl+arom }}\right), 7.48-7.53(\mathrm{~m}, 4 \mathrm{H}$, $\left.\mathrm{H}_{\text {arom+naph }}\right), 7.57\left(\mathrm{~d}, 1 \mathrm{H}, J=16.2 \mathrm{~Hz}, \mathrm{H}_{\text {vinyl }}\right), 7.77-7.87(\mathrm{~m}, 4 \mathrm{H}$, $\left.\mathrm{H}_{\text {naph }}\right), 7.94\left(\mathrm{~s}, 1 \mathrm{H}, \mathrm{H}_{\text {naph }}\right) .{ }^{13} \mathrm{C} \mathrm{NMR}\left(\mathrm{CDCl}_{3}, 75 \mathrm{MHz}\right): \delta 97.1$ (C), $111.3(\mathrm{CH}), 114.0(\mathrm{CH}), 119.7(\mathrm{CH}), 123.4(\mathrm{CH}), 123.7$ $(\mathrm{CH}), 126.0(\mathrm{CH}), 126.6(\mathrm{CH}), 126.7(\mathrm{CH}), 127.9(2 \mathrm{CH}), 128.4$ $(\mathrm{CH}), 128.7(\mathrm{CH}), 129.0(\mathrm{C}), 132.0(\mathrm{CH}), 133.6(\mathrm{C}), 133.8(\mathrm{C})$, 134.0 (C), $151.6(\mathrm{C}), 153.8(\mathrm{C})$. IR $\left(v \mathrm{~cm}^{-1}\right)$ : 804, 962, 1015, 1195, 1258, 1446. MS (APCI+) $m / z 349.0(\mathrm{M}+\mathrm{H})^{+}\left({ }^{79} \mathrm{Br}\right), 351.0$ $(\mathrm{M}+\mathrm{H})^{+}\left({ }^{81} \mathrm{Br}\right)$. Anal. $\mathrm{C}_{20} \mathrm{H}_{13} \mathrm{BrO}$ (349.22): found $\mathrm{C} 68.55, \mathrm{H}$ 3.51 requires $\mathrm{C} 68.79, \mathrm{H} 3.75$.

(E)-3-Bromo-2-(2-(thiophen-3-yl)vinyl)benzofuran (2g) (171 $\mathrm{mg} ; 56 \%)$. Gray solid. Mp 65-66 ${ }^{\circ} \mathrm{C} .{ }^{1} \mathrm{H} \mathrm{NMR}\left(\mathrm{CDCl}_{3}, 300\right.$ $\mathrm{MHz}): 6.95\left(\mathrm{~d}, 1 \mathrm{H}, J=16.1 \mathrm{~Hz}, \mathrm{H}_{\text {vinyl }}\right), 7.29-7.34(\mathrm{~m}, 2 \mathrm{H}$, $\mathrm{H}_{\text {vinyl+arom }}$ ), 7.35-7.47 (m, 5H, $\left.\mathrm{H}_{\text {arom }}\right), 7.48-7.51\left(\mathrm{~m}, 1 \mathrm{H}, \mathrm{H}_{\text {arom }}\right)$. ${ }^{13} \mathrm{C}$ NMR $\left(\mathrm{CDCl}_{3}, 75 \mathrm{MHz}\right): \delta 96.6(\mathrm{C}), 111.2(\mathrm{CH}), 113.7$ $(\mathrm{CH}), 119.5(\mathrm{CH}), 123.6(\mathrm{CH}), 124.5(\mathrm{CH}), 125.0(\mathrm{CH}), 125.8$ $(\mathrm{CH}), 126.0(\mathrm{CH}), 126.7(\mathrm{CH}), 129.0(\mathrm{C}), 139.4(\mathrm{C}), 151.4(\mathrm{C})$, $153.6(\mathrm{C})$. IR $\left(v^{-1} \mathrm{~cm}^{-1}\right): 738,823,853,941,1014,1191,1257$, 1404, 1445, 1632. MS (APCI+) $\mathrm{m} / z, 305.0(\mathrm{M}+\mathrm{H})^{+}\left({ }^{79} \mathrm{Br}\right), 307.0$ $(\mathrm{M}+\mathrm{H})^{+}\left({ }^{81} \mathrm{Br}\right)$. Anal. $\mathrm{C}_{14} \mathrm{H}_{9} \mathrm{BrOS}$ (305.19): found $\mathrm{C} 55.19, \mathrm{H}$ 3.06. requires C 55.10, H 2.97 .

3-Bromo-2-(2-methylprop-1-enyl)benzofuran (2h) (128 mg; $51 \%) . \mathrm{R}_{\mathrm{f}}=0.62\left(\right.$ Cyclohexane/ $\left.\mathrm{CH}_{2} \mathrm{Cl}_{2}: 95 / 5\right) . \mathrm{NMR}{ }^{1} \mathrm{H}\left(\mathrm{CDCl}_{3}\right.$, $300 \mathrm{MHz}): \delta 2.01\left(\mathrm{~s}, 3 \mathrm{H}, \mathrm{CH}_{3}\right), 2.22\left(\mathrm{~d}, 3 \mathrm{H}, J=1.3 \mathrm{~Hz}, \mathrm{CH}_{3}\right)$, $6.25\left(\mathrm{q}, J=1.3 \mathrm{~Hz}, 1 \mathrm{H}, \mathrm{H}_{\text {vinyl }}\right), 7.26-7.33$ (m, $\left.2 \mathrm{H}, \mathrm{H}_{\text {arom }}\right), 741-$ $7.50\left(\mathrm{~m}, 2 \mathrm{H}, \mathrm{H}_{\text {arom }}\right) . \mathrm{NMR}{ }^{13} \mathrm{C}\left(\mathrm{CDCl}_{3}, 75 \mathrm{MHz}\right): \delta 20.8\left(\mathrm{CH}_{3}\right)$, $27.8\left(\mathrm{CH}_{3}\right), 95.0(\mathrm{C}), 111.2,111.5(\mathrm{CH}), 119.3(\mathrm{CH}), 123.4$ $(\mathrm{CH}), 124.9(\mathrm{CH}), 128.3(\mathrm{C}), 141.8(\mathrm{C}), 152.1(\mathrm{C}), 153.4$. IR ( $v$ $\mathrm{cm}^{-1}$ ): 2930, 1450, 1259, 1197, 1011, 738. MS (APCI+) 252.0 $\left([\mathrm{M}+\mathrm{H}]^{+}\right)$. Anal. $\mathrm{C}_{12} \mathrm{H}_{11} \mathrm{BrO}$ (251.12): found C 57.33, H 4.37 requires C 57.39, $\mathrm{H} 4.42$.

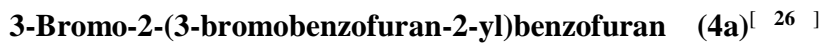
(376 mg; 96\%).

White solid. Mp 224-225 ${ }^{\circ} \mathrm{C}$. $\mathrm{R}_{\mathrm{f}}=0.43$ (Cyclohexane/ $\mathrm{CH}_{2} \mathrm{Cl}_{2}$ : 96/4). NMR ${ }^{1} \mathrm{H}\left(\mathrm{CDCl}_{3}, 400 \mathrm{MHz}\right): \delta 7.38(\mathrm{t}, J=7.4 \mathrm{~Hz}, 2 \mathrm{H}$, $\left.\mathrm{H}_{\text {arom }}\right), 7.45$ (t, $\left.J=7.4 \mathrm{~Hz}, 2 \mathrm{H}, \mathrm{H}_{\text {arom }}\right), 7.59$ (d, $J=8.2 \mathrm{~Hz}, 2 \mathrm{H}$, $\left.\mathrm{H}_{\text {arom }}\right), 7.64\left(\mathrm{~d}, J=7.7 \mathrm{~Hz}, 2 \mathrm{H}, \mathrm{H}_{\text {arom }}\right) . \mathrm{RMN}{ }^{13} \mathrm{C}\left(\mathrm{CDCl}_{3}, 100\right.$ MHz): $\delta 98.3$ (2C), 111.9 (2C), 120.5 (2C), 124.1 (2C), 126.9 (2C), 128.7 (2C), 142.8 (2C), 154.7 (2C). IR ( $\left.v \mathrm{~cm}^{-1}\right)$ : 2923, 1437, 1260, 1211, 1122, 743. MS (APCI+) m/z 312.0 ([M$\left.\left.{ }^{81} \mathrm{Br}+\mathrm{H}\right]^{+}\right), 314.0\left(\left[\mathrm{M}^{79} \mathrm{Br}+\mathrm{H}\right]^{+}\right)$.

\section{3-Bromo-2-(3-bromobenzo[b]thiophen-2-yl)benzo[b]thio-} phene (4b) ${ }^{[27]}$ (395 mg; 97\%). Yellow solid. $\mathrm{R}_{\mathrm{f}}=0.67$ (Cyclohexane/ $\mathrm{CH}_{2} \mathrm{Cl}_{2}$ : 9/1). NMR ${ }^{1} \mathrm{H}\left(\mathrm{CDCl}_{3}, 300 \mathrm{MHz}\right)$ : $\delta 7.50$ (m, 4H, $\left.\mathrm{H}_{\text {arom }}\right), 7.85$ (d, $\left.J=7.6 \mathrm{~Hz}, 2 \mathrm{H}, \mathrm{H}_{\text {arom }}\right), 7.93$ (d, $J$ $\left.=7.7 \mathrm{~Hz}, 2 \mathrm{H}, \mathrm{H}_{\text {arom }}\right) . \mathrm{NMR}{ }^{13} \mathrm{C}\left(\mathrm{CDCl}_{3}, 75 \mathrm{MHz}\right): \delta 111.0(2 \mathrm{C})$, 122.4 (2C), 124.2 (2C), 125.6 (2C), 126,5 (2C), 129.5 (2C), 138.2 (2C), 139.3 (2C).

3-Bromo-2-(3-bromobenzothiophen-2-yl)benzofuran (4c) (99\%). Yellow solid. $\mathrm{Mp} 113-118 \quad{ }^{\circ} \mathrm{C} . \quad \mathrm{R}_{\mathrm{f}}=0.59$ (Cyclohexane/EtOAc: 96/4). NMR ${ }^{1} \mathrm{H}\left(\mathrm{CDCl}_{3}, 300 \mathrm{MHz}\right): \delta$ 7.35-7.64 (m, 6H, $\left.\mathrm{H}_{\text {arom }}\right), 7.86\left(\mathrm{~d}, J=7.5 \mathrm{~Hz}, 1 \mathrm{H}, \mathrm{H}_{\text {arom }}\right), 7.96$ (d, $\left.J=8.4 \mathrm{~Hz}, 1 \mathrm{H}, \mathrm{H}_{\text {arom }}\right)$. NMR ${ }^{13} \mathrm{C}\left(\mathrm{CDCl}_{3}, 75 \mathrm{MHz}\right): \delta 99.3$ (C), $109.9(\mathrm{C}), 111.8(\mathrm{CH}), 120.4(\mathrm{CH}), 122.4(\mathrm{CH}), 124.0(\mathrm{CH})$, $124.3(\mathrm{CH}), 125.6(\mathrm{CH}), 126.7(2 \mathrm{CH}), 128.7(\mathrm{C}), 138.3(\mathrm{C})$, 138.8 (C), $145.4(\mathrm{C}), 148.4(\mathrm{C}), 154.2(\mathrm{C})$. IR $\left(v \mathrm{~cm}^{-1}\right): 2923$, 1454, 1257, 1241, 1040, 978, 737, 720. MS (APCI +) $m / z, 409.0$ $\left([\mathrm{M}+\mathrm{H}]^{+}\right)$. Anal. $\mathrm{C}_{16} \mathrm{H}_{8} \mathrm{Br}_{2} \mathrm{OS}$ (408.11): found C 46.89, H 1.89 requires $\mathrm{C}$ 47.09, $\mathrm{H} 1.98$.

3-Bromo-2-(2-(4-methoxyphenyl)ethynyl)benzofuran (4d) (288 mg; 88\%). Yellow solid. Mp 82-83 ${ }^{\circ} \mathrm{C} . \quad \mathrm{R}_{\mathrm{f}}=0.23$ (Cyclohexane/ $\mathrm{CH}_{2} \mathrm{Cl}_{2}$ : 9/1). NMR ${ }^{1} \mathrm{H}\left(\mathrm{CDCl}_{3}, 300 \mathrm{MHz}\right)$ : $\delta 3.83\left(\mathrm{~s}, 3 \mathrm{H}, \mathrm{OCH}_{3}\right), 6.90\left(\mathrm{~d}, J=7.6 \mathrm{~Hz}, 2 \mathrm{H}, \mathrm{H}_{\text {arom }}\right), 7.29-7,41$ $\left(\mathrm{m}, 2 \mathrm{H}, \mathrm{H}_{\text {arom }}\right), 7.45\left(\mathrm{~d}, J=8.3 \mathrm{~Hz}, 1 \mathrm{H}, \mathrm{H}_{\text {arom }}\right), 7.50(\mathrm{~d}, J=7.4$ $\left.\mathrm{Hz}, 1 \mathrm{H}, \mathrm{H}_{\text {arom }}\right), 7.56\left(\mathrm{~d}, J=7.6 \mathrm{~Hz}, 2 \mathrm{H}, \mathrm{H}_{\text {arom }}\right) . \mathrm{NMR}{ }^{13} \mathrm{C}$ $\left(\mathrm{CDCl}_{3}, 75 \mathrm{MHz}\right): \delta 55.5\left(\mathrm{OCH}_{3}\right) ; 77.0(\mathrm{C}), 99.5(\mathrm{C}), 102.9(\mathrm{C})$, $111.6(\mathrm{CH}), 113.6(\mathrm{C}), 114.3(2 \mathrm{CH}), 120.0(\mathrm{CH}), 123.9(\mathrm{CH})$, $126.6(\mathrm{CH}), 127.8(\mathrm{C}), 133.6(2 \mathrm{CH}), 137.8(\mathrm{C}), 153.8(\mathrm{C})$, 160.7 (C). IR $\left(v^{-1}\right): 2208,1504,1290,1249,1016,905,830$, 725. MS (APCI+) $m / z \quad 328.0\left([\mathrm{M}+\mathrm{H}]^{+}\right)$. Anal. $\mathrm{C}_{17} \mathrm{H}_{11} \mathrm{BrO}_{2}$ (327.17): found C 62.13, H 3.10 requires C 62.41, H 3.39.

(E)-3-Bromo-2-(2-(3-bromobenzofuran-2-yl)vinyl)benzofuran (6) $(92 \mathrm{mg} ; 21 \%)$. Yellow solid. Mp 232-233 ${ }^{\circ} \mathrm{C}$. NMR ${ }^{1} \mathrm{H}\left(\mathrm{CDCl}_{3}, 300 \mathrm{MHz}\right): \delta 7.26\left(\mathrm{~s}, 2 \mathrm{H}, \mathrm{H}_{\text {vinyl }}\right), 7.40\left(\mathrm{~m}, 2 \mathrm{H}, \mathrm{H}_{\text {arom }}\right)$, $7.49\left(\mathrm{~m}, 2 \mathrm{H}, \mathrm{H}_{\text {arom }}\right), 7.56$ (dd, $\left.2 \mathrm{H}, J=7.7,0.8 \mathrm{~Hz}, \mathrm{H}_{\text {arom }}\right), 7.73$ $\left(\mathrm{d}, 2 \mathrm{H}, J=8.2 \mathrm{~Hz}, \mathrm{H}_{\text {arom }}\right)$. $\mathrm{NMR}{ }^{13} \mathrm{C}\left(\mathrm{CDCl}_{3}, 75 \mathrm{MHz}\right): \delta 99.3$ $(\mathrm{CH}), 115.5(\mathrm{CH}), 116.0(\mathrm{CH}), 120.0(\mathrm{CH}), 123.9(\mathrm{CH}), 126.7$ $(\mathrm{CH}), 129.0(\mathrm{C}), 150.9(\mathrm{C}), 154.1(\mathrm{C})$. IR $\left(v_{\mathrm{cm}}^{-1}\right)$ : 738, 861, 943, 1017, 1106, 1202, 1259, 1343, 1447, 1612. MS (APCI+) $m / z$ 441.0 $\left([\mathrm{M}+\mathrm{Na}]^{+}\right)$. Anal. $\mathrm{C}_{18} \mathrm{H}_{10} \mathrm{Br}_{2} \mathrm{O}_{2}$ (418.08): found $\mathrm{C}$ 51.50, H 2.28 requires C 51.71, H 2.41.

3-Bromo-2-(4-methoxyphenyl)benzofuran (8a) ${ }^{[28]}$ (297 mg; 98\%). Yellow solid. Mp 66-67 ${ }^{\circ} \mathrm{C} . \mathrm{R}_{\mathrm{f}}=0.51$ (Cyclohexane/ $\left.\mathrm{CH}_{2} \mathrm{Cl}_{2}: 8 / 2\right)$. NMR ${ }^{1} \mathrm{H}\left(\mathrm{CDCl}_{3}, 400 \mathrm{MHz}\right): \delta 3.88(\mathrm{~d}, J=0.9 \mathrm{~Hz}$, $\left.3 \mathrm{H}, \mathrm{OCH}_{3}\right), 7.02\left(\mathrm{~d}, J=7.9 \mathrm{~Hz}, 2 \mathrm{H}, \mathrm{H}_{\text {arom }}\right), 7.32\left(\mathrm{~m}, 2 \mathrm{H}, \mathrm{H}_{\text {arom }}\right)$, $7.50\left(\mathrm{~m}, 2 \mathrm{H}, \mathrm{H}_{\text {arom }}\right), 8.12\left(\mathrm{~d}, J=7.9 \mathrm{~Hz}, 2 \mathrm{H}, \mathrm{H}_{\text {arom }}\right) . \mathrm{NMR}{ }^{13} \mathrm{C}$ $\left(\mathrm{CDCl}_{3}, 100 \mathrm{MHz}\right): \delta 55.5\left(\mathrm{OCH}_{3}\right), 92.3,111.3,114.2$, (2), $119.7,122.4,123.5,125.3,128.5$ (2), 129.9, 150.7, 153.1, 160.4. IR $\left(v \mathrm{~cm}^{-1}\right): 2959,1609,1450,1251,1117,1072,1030,985$, 830, 783. MS (APCI+) $m / z, 224\left([\mathrm{M}-\mathrm{Br}+\mathrm{H}]^{+}\right.$.

3-Bromo-2-(2-methoxyphenyl)benzofuran $(\mathbf{8 b})^{[\mathbf{9 b}]}(239 \mathrm{mg}$; $79 \%) . \mathrm{R}_{\mathrm{f}}=0.38\left(\right.$ Cyclohexane/ $\left.\mathrm{CH}_{2} \mathrm{Cl}_{2}: 8 / 2\right) . \mathrm{NMR}{ }^{1} \mathrm{H}\left(\mathrm{CDCl}_{3}\right.$, $300 \mathrm{MHz}$ ): $\delta 3.89$ (s, 3H, OCH 3$), 7.05$ (d, $\left.J=8.1 \mathrm{~Hz}, 1 \mathrm{H}, \mathrm{H}_{\text {arom }}\right)$, $7.09\left(\mathrm{td}, J=7.5 \mathrm{~Hz}, J=1.0 \mathrm{~Hz}, 1 \mathrm{H}, \mathrm{H}_{\text {arom }}\right), 7.35$ (m, $\left.2 \mathrm{H}, \mathrm{H}_{\text {arom }}\right)$, $7.49\left(\mathrm{~m}, 2 \mathrm{H}, \mathrm{H}_{\text {arom }}\right), 7.60\left(\mathrm{~m}, 2 \mathrm{H}, \mathrm{H}_{\text {arom }}\right) . \mathrm{NMR}{ }^{13} \mathrm{C}\left(\mathrm{CDCl}_{3}, 75\right.$ $\mathrm{MHz}): \delta 55.8\left(\mathrm{OCH}_{3}\right), 96.8(\mathrm{C}), 111.6(\mathrm{CH}), 111.7(\mathrm{CH}), 118.4$ (C), $119.9(\mathrm{CH}), 120.6(\mathrm{CH}), 123.3(\mathrm{CH}), 125.3(\mathrm{CH}), 129.1$ $(\mathrm{CH}), 131.5(\mathrm{CH}), 131.8(\mathrm{CH}), 150.6(\mathrm{C}), 154.0(\mathrm{C}), 157.8(\mathrm{C})$. IR $\left(v^{-1}\right): 2933,1486,1447,1255,1058,1044,1024,740$. MS (ESI+) $m / z, 224.0\left([\mathrm{M}-\mathrm{Br}+\mathrm{H}]^{+}\right)$. Anal. $\mathrm{C}_{15} \mathrm{H}_{11} \mathrm{BrO}_{2}(303.15)$ : found C 59.19, H 3.76 requires C 59.43, H 3.66.

3-Bromo-2-p-tolylbenzofuran (8c) (229 mg; 80\%). Yellow solid. Mp 70-72 ${ }^{\circ} \mathrm{C}$. $\mathrm{R}_{\mathrm{f}}=0.65$ (Cyclohexane/ $\mathrm{CH}_{2} \mathrm{Cl}_{2}$ : 9/1). NMR ${ }^{1} \mathrm{H}\left(\mathrm{CDCl}_{3}, 400 \mathrm{MHz}\right): \delta 2.43\left(\mathrm{~s}, 3 \mathrm{H}, \mathrm{CH}_{3}\right), 7.32(\mathrm{~m}, 4 \mathrm{H}$, $\left.\mathrm{H}_{\text {arom }}\right), 7.53\left(\mathrm{~m}, 2 \mathrm{H}, \mathrm{H}_{\text {arom }}\right), 8.07$ (d, $\left.J=8.3 \mathrm{~Hz}, 2 \mathrm{H}, \mathrm{H}_{\text {arom }}\right)$. $\mathrm{NMR}{ }^{13} \mathrm{C}\left(\mathrm{CDCl}_{3}, 100 \mathrm{MHz}\right) \delta: 21.6\left(\mathrm{CH}_{3}\right), 93.3,111.3,119.9$ 123.5, 125.5, 126.9 (3), 129.4 (2C), 129.8, 139.4, 150.8, 153.2. IR $\left(v \mathrm{~cm}^{-1}\right): 2919,1503,1450,1253,1204,1072,1020,817$, 741. MS (APCI+) $m / z 208\left([\mathrm{M}-\mathrm{Br}+\mathrm{H}]^{+}\right)$.

4-(3-Bromobenzofuran-2-yl)- $\boldsymbol{N}, \boldsymbol{N}$-dimethylbenzenamine (8d) $(221 \mathrm{mg} ; 74 \%) . \mathrm{R}_{\mathrm{f}}=0.57\left(\right.$ Cyclohexane/ $\left.\mathrm{CH}_{2} \mathrm{Cl}_{2}: 8 / 2\right) . \mathrm{NMR}{ }^{1} \mathrm{H}$ $\left(\mathrm{CDCl}_{3}, 300 \mathrm{MHz}\right): \delta 3.04\left(\mathrm{~s}, 6 \mathrm{H}, \mathrm{N}-\mathrm{CH}_{3}\right), 6.80(\mathrm{~d}, J=8.9 \mathrm{~Hz}$, 
$2 \mathrm{H},), 7.29\left(\mathrm{~m}, 2 \mathrm{H}, \mathrm{H}_{\text {arom }}\right), 7.50\left(\mathrm{~m}, 2 \mathrm{H}, \mathrm{H}_{\text {arom }}\right), 8.08(\mathrm{~d}, J=8.7$ $\mathrm{Hz}, 2 \mathrm{H},) . \mathrm{NMR}{ }^{13} \mathrm{C}\left(\mathrm{CDCl}_{3}, 75 \mathrm{MHz}\right): \delta 40.3\left(2 \mathrm{CH}_{3}\right), 90.6$, $111.0(\mathrm{CH}), 111.9(2 \mathrm{C}), 117.4(\mathrm{C}), 119.3(\mathrm{CH}), 123.3(\mathrm{CH})$, $124.6(\mathrm{CH}), 128.1$ (2 C), $130.2(\mathrm{C}), 150.8(\mathrm{C}), 151.6(\mathrm{C}), 153.0$ (C). IR $\left(v \mathrm{~cm}^{-1}\right)$ : 2889, 1607, 1510, 1450, 1361, 1194, 816, 742. $\mathrm{SM}(\mathrm{APCI}+) \mathrm{m} / z \quad 316\left(\left[\mathrm{M}\left({ }^{79} \mathrm{Br}\right)+\mathrm{H}\right]^{+}\right), 318\left(\left[\mathrm{M}\left({ }^{81} \mathrm{Br}\right)+\mathrm{H}\right]^{+}\right)$. Anal. $\mathrm{C}_{16} \mathrm{H}_{14} \mathrm{BrNO}$ (299.16): found $\mathrm{C} 60.54, \mathrm{H} 4.29, \mathrm{~N} 4.23$ requires $\mathrm{C} 60.78, \mathrm{H} 4.46, \mathrm{~N} 4.43$.

3-Bromo-2-(naphthalen-1-yl)benzofuran (8e) (242 mg; 75\%). White solid. Mp 107-109 ${ }^{\circ} \mathrm{C}$. $\mathrm{R}_{\mathrm{f}}=0.65$ (Cyclohexane/ $\mathrm{CH}_{2} \mathrm{Cl}_{2}$ : 9/1). NMR ${ }^{1} \mathrm{H}\left(\mathrm{CDCl}_{3}, 300 \mathrm{MHz}\right): \delta 7.43(\mathrm{~m}, 2 \mathrm{H}),, 7.62(\mathrm{~m}$, $5 \mathrm{H}), 7.87\left(\mathrm{~d}, J=8.7 \mathrm{~Hz}, 1 \mathrm{H}, \mathrm{H}_{\text {naph }}\right), 8.00(\mathrm{~m}, 3 \mathrm{H}) . \mathrm{NMR}{ }^{13} \mathrm{C}$ $\left(\mathrm{CDCl}_{3}, 75 \mathrm{MHz}\right): \delta 97.3,111.7,(\mathrm{CH}), 120.1(\mathrm{CH}), 123.7(\mathrm{CH})$, $125.1(\mathrm{C}), 125.7(\mathrm{CH}), 126.1(\mathrm{CH}), 126.4(\mathrm{CH}), 126.5(\mathrm{C})$, $127.0(\mathrm{CH}), 128.6,(\mathrm{CH}), 128.9(\mathrm{C}), 129.7(\mathrm{CH}), 130.6(\mathrm{CH})$, 131.6 (C), 133.9 (C), 152.2 (C), 154.1 (C). IR $\left(v \mathrm{~cm}^{-1}\right)$ : 3053 , 1449, 1259, 1029, 1014, 968, 800, 772, 743. MS (APCI+) $\mathrm{m} / \mathrm{z}$ $244.0\left([\mathrm{M}-\mathrm{Br}+\mathrm{H}]^{+}\right)$. Anal. $\mathrm{C}_{18} \mathrm{H}_{11} \mathrm{BrO}$ (323.18): found $\mathrm{C} 66.78$, H 3.36 requires $\mathrm{C} 66.89, \mathrm{H} 3.43$;

3-Bromo-2-(naphthalen-2-yl)benzofuran (8f) (197 mg; 61\%). Yellow solid. Mp 98-102 ${ }^{\circ} \mathrm{C}$. $\mathrm{R}_{\mathrm{f}}=0.56\left(\right.$ Cyclohexane/ $\mathrm{CH}_{2} \mathrm{Cl}_{2}$ : 9/1). $\mathrm{RMN}{ }^{1} \mathrm{H}\left(\mathrm{CDCl}_{3}, 300 \mathrm{MHz}\right): \delta 7.38(\mathrm{~m}, 2 \mathrm{H}),, 7.57(\mathrm{~m}$, $4 \mathrm{H}), 7.87(\mathrm{~m}, 4 \mathrm{H}), 7.96(\mathrm{~m}, 2 \mathrm{H}), 8.30\left(\mathrm{~d}, J=8.7 \mathrm{~Hz}, 1 \mathrm{H}, \mathrm{H}_{\text {naph }}\right)$, $8.68\left(\mathrm{~s}, 1 \mathrm{H}, \mathrm{H}_{\text {naph }}\right) . \mathrm{NMR}{ }^{13} \mathrm{C}\left(\mathrm{CDCl}_{3}, 75 \mathrm{MHz}\right): \delta 94.4(\mathrm{C})$, $110.1(\mathrm{C}), 111.4(\mathrm{CH}), 120.1(\mathrm{CH}), 123.7(\mathrm{CH}), 124.0(\mathrm{CH})$, $125.8(\mathrm{CH}), 126.6(\mathrm{CH}), 126.8(\mathrm{CH}), 127.1(\mathrm{CH}), 127.9(\mathrm{CH})$, $128.4(\mathrm{CH}), 128.8(\mathrm{CH}), 129.8(\mathrm{C}), 133.2(\mathrm{C}), 133.4(\mathrm{C}), 150.5$ (C), 153.4 (C). IR $\left(v^{-1}\right)$ : 3058, 1450, 1259, 1065, 994, 857, 815, 742. MS (APCI+) $m / z$ 244.0 $\left([\mathrm{M}-\mathrm{Br}+\mathrm{H}]^{+}\right)$. Anal. $\mathrm{C}_{18} \mathrm{H}_{11} \mathrm{BrO}$ (323.18): found $\mathrm{C} 67.01, \mathrm{H} 3.60$ requires $\mathrm{C} 66.89, \mathrm{H}$ 3.43 .

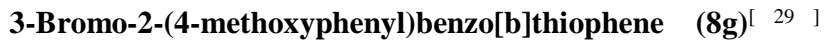
(309 mg; 97\%).

Yellow solid. Mp $83{ }^{\circ} \mathrm{C}$. $\mathrm{R}_{\mathrm{f}}=0.41$ (Cyclohexane/ $\left.\mathrm{CH}_{2} \mathrm{Cl}_{2}: 8 / 2\right)$. $\mathrm{NMR}{ }^{1} \mathrm{H}\left(\mathrm{CDCl}_{3}, 300 \mathrm{MHz}\right): \delta 3.88\left(\mathrm{~s}, 3 \mathrm{H}, \mathrm{OCH}_{3}\right), 7.02(\mathrm{~d}, J=$ $\left.8.5 \mathrm{~Hz}, 2 \mathrm{H}, \mathrm{H}_{\text {arom }}\right), 7.39$ (t, $\left.J=7.5 \mathrm{~Hz}, 1 \mathrm{H}, \mathrm{H}_{\text {arom }}\right), 7.47$ (t, $J=$ $\left.7.6 \mathrm{~Hz}, 1 \mathrm{H}, \mathrm{H}_{\text {arom }}\right), 7.71\left(\mathrm{~d}, J=8.4 \mathrm{~Hz}, 2 \mathrm{H}, \mathrm{H}_{\text {arom }}\right), 7.80$ (d, $J=$ $\left.7.8 \mathrm{~Hz}, 1 \mathrm{H}, \mathrm{H}_{\text {arom }}\right), 7.85$ (d, $J=8.0 \mathrm{~Hz}, 1 \mathrm{H}, \mathrm{H}_{\text {arom }}$ ). NMR ${ }^{13} \mathrm{C}$ $\left(\mathrm{CDCl}_{3}, 75 \mathrm{MHz}\right): \delta 55.5\left(\mathrm{OCH}_{3}\right), 104.4(\mathrm{C}), 114.2(2 \mathrm{C}), 122.2$ $(\mathrm{CH}), 123.6(\mathrm{CH}), 125.3(\mathrm{CH}), 125.4(\mathrm{CH}), 125.5(\mathrm{C}), 131.1(2$ C), 137.6 (C), 138.3 (C), $139.4(\mathrm{C}), 160.2(\mathrm{C})$. IR $\left(\mathrm{v} \mathrm{cm}^{-1}\right)$ : 2922, $1606,1494,1433,1248,1177,1032,831,802,749$. MS $(\mathrm{APCI}+) \mathrm{m} / z, 240\left([\mathrm{M}-\mathrm{Br}+\mathrm{H}]^{+}\right)$.

\section{3-Bromo-2-(2-methoxyphenyl)benzo[b]thiophene (8h) (258 $\mathrm{mg} ; 81 \%)$. \\ Beige solid. Mp 85-87 ${ }^{\circ} \mathrm{C} . \mathrm{R}_{\mathrm{f}}=0.27$ (Cyclohexane). NMR ${ }^{1} \mathrm{H}$ $\left(\mathrm{CDCl}_{3}, 300 \mathrm{MHz}\right): \delta 3.86\left(\mathrm{~s}, 3 \mathrm{H}, \mathrm{OCH}_{3}\right), 7.06\left(\mathrm{~m}, 2 \mathrm{H}, \mathrm{H}_{\text {arom }}\right)$, $7.45\left(\mathrm{~m}, 4 \mathrm{H}, \mathrm{H}_{\text {arom }}\right), 7.82\left(\mathrm{~d}, J=7.7 \mathrm{~Hz}, 1 \mathrm{H}, \mathrm{H}_{\text {arom }}\right), 7.87$ (d, $J=$ $\left.8.0 \mathrm{~Hz}, 1 \mathrm{H}, \mathrm{H}_{\text {arom }}\right) . \mathrm{NMR}{ }^{13} \mathrm{C}\left(\mathrm{CDCl}_{3}, 75 \mathrm{MHz}\right): \delta 55.8\left(\mathrm{OCH}_{3}\right)$, $107.8(\mathrm{C}), 111.6(\mathrm{CH}), 120.6(\mathrm{CH}), 121.9(\mathrm{C}), 122.3(\mathrm{CH})$, $123.5(\mathrm{CH}), 125.0(\mathrm{CH}), 125.3(\mathrm{CH}), 130.8(\mathrm{CH}), 132.5(\mathrm{C})$, 135.4 (C), 138.5 (C), 138.7 (C), 157,4 (C). IR ( $\left.\mathrm{c} \mathrm{cm}^{-1}\right)$ : 2938, $1482,1459,1432,1248,1115,1024,889,748,725$. MS (APCI+) $m / z 240\left([\mathrm{M}-\mathrm{Br}+\mathrm{H}]^{+}\right)$. Anal. $\mathrm{C}_{15} \mathrm{H}_{11} \mathrm{BrOS}(319.22)$ : found C 56.29, H 3.36 requires C 56.44, H 3.47.}

4-Bromo-3-(2-methoxyphenyl)-1H-isochromen-1-one (8i) (324 mg; 98\%). White solid. Mp 129-131 ${ }^{\circ} \mathrm{C} . \quad \mathrm{R}_{\mathrm{f}}=0.37$ (Cyclohexane/ $\left.\mathrm{CH}_{2} \mathrm{Cl}_{2}: 8 / 2\right)$. NMR ${ }^{1} \mathrm{H}\left(\mathrm{CDCl}_{3}, 300 \mathrm{MHz}\right): \delta$ $3.85\left(\mathrm{~s}, 3 \mathrm{H}, \mathrm{OCH}_{3}\right), 7.00\left(\mathrm{~d}, J=8.4 \mathrm{~Hz}, 1 \mathrm{H}, \mathrm{H}_{\text {arom }}\right), 7.05(\mathrm{td}, J=$ $\left.7.5, J=0.9 \mathrm{~Hz}, 1 \mathrm{H}, \mathrm{H}_{\text {arom }}\right), 7.40-7.49\left(\mathrm{~m}, 2 \mathrm{H}, \mathrm{H}_{\text {arom }}\right), 7.60$ (td, $J$ $\left.=7.5 \mathrm{~Hz}, J=1.2 \mathrm{~Hz}, 1 \mathrm{H}, \mathrm{H}_{\text {arom }}\right), 7.84(\mathrm{td}, J=7.6 \mathrm{~Hz}, J=1.3 \mathrm{~Hz}$, $\left.1 \mathrm{H}, \mathrm{H}_{\text {arom }}\right), 7.92\left(\mathrm{~d}, J=8.1 \mathrm{~Hz}, 1 \mathrm{H}, \mathrm{H}_{\text {arom }}\right), 8.35(\mathrm{dd}, J=7.9 \mathrm{~Hz}$, $\left.J=0.8 \mathrm{~Hz}, 1 \mathrm{H}, \mathrm{H}_{\text {arom }}\right)$. NMR ${ }^{13} \mathrm{C}\left(\mathrm{CDCl}_{3}, 75 \mathrm{MHz}\right): \delta 55.8$ $\left(\mathrm{OCH}_{3}\right), 103.9(\mathrm{C}), 111.5(\mathrm{CH}), 120.4(\mathrm{CH}), 121.0(\mathrm{C}), 122.5$ (C), 126.5, CH), $129.2(\mathrm{CH}), 130.0(\mathrm{CH}), 131.3(\mathrm{CH}), 131.9$ $(\mathrm{CH}), 135.4(\mathrm{CH}), 136.6(\mathrm{C}), 150.8(\mathrm{C}), 157.5(\mathrm{C}), 161.7$ (C). IR $\left(v \mathrm{~cm}^{-1}\right): 2952,1732,1279,1072,1020,753$. MS (ESI+) $\mathrm{m} / \mathrm{z}$ $354\left([\mathrm{M}+\mathrm{Na}]^{+}\right)$. Anal. $\mathrm{C}_{16} \mathrm{H}_{11} \mathrm{BrO}_{3}$ (329.99): found $\mathrm{C} 57.88, \mathrm{H}$ 3.20 requires C 58.03, H 3.35 .

(2-(3-Bromobenzofuran-2-yl)phenoxy)(tert-butyl)dimethylsilane (8j) $(375 \mathrm{mg} ; 93 \%) . \mathrm{R}_{\mathrm{f}}=0.76\left(\right.$ Cyclohexane/ $\mathrm{CH}_{2} \mathrm{Cl}_{2}$ : 9/1). NMR ${ }^{1} \mathrm{H}\left(\mathrm{CDCl}_{3}, 300 \mathrm{MHz}\right): \delta 0.06\left(\mathrm{~s}, 6 \mathrm{H}, \mathrm{CH}_{3} \mathrm{Si}\right), 0.84$ (s, 9H, $\left.\mathrm{CH}_{3} \mathrm{C}\right), 6.99$ (d, $\left.J=8.2 \mathrm{~Hz}, 1 \mathrm{H}, \mathrm{H}_{\text {arom }}\right), 7.09$ (td, $J=7.5$ $\left.\mathrm{Hz}, J=1.0 \mathrm{~Hz}, 1 \mathrm{H}, \mathrm{H}_{\text {arom }}\right), 7.36\left(\mathrm{~m}, 3 \mathrm{H}, \mathrm{H}_{\text {arom }}\right), 7.48(\mathrm{~m}, 1 \mathrm{H}$, $\left.\mathrm{H}_{\text {arom }}\right), 7.59\left(\mathrm{~m}, 2 \mathrm{H}, \mathrm{H}_{\text {arom }}\right)$. $\mathrm{NMR}{ }^{13} \mathrm{C}\left(\mathrm{CDCl}_{3}, 100 \mathrm{MHz}\right): \delta-4.5$ $\left(2 \mathrm{CH}_{3}\right), 18.2(\mathrm{C}), 25.6\left(3 \mathrm{CH}_{3}\right), 96.3(\mathrm{C}), 111.5(\mathrm{CH}), 119.8$ $(\mathrm{CH}), 120.8(\mathrm{CH}), 121.3(\mathrm{CH}), 123.3(\mathrm{CH}), 125.3(\mathrm{CH}), 128.8$ (2 C), $131.3(\mathrm{CH}), 132.0(\mathrm{CH}), 151.2(\mathrm{C}), 153.8(\mathrm{C}), 154.4(\mathrm{C})$. IR $\left(v^{-1} \mathrm{~cm}^{-1}\right)$ : 2930, 1483, 1449, 1281, 1258, 916, 887, 826, 780, 743. MS (APCI+) $m / z, 403\left(\left[\mathrm{M}\left({ }^{79} \mathrm{Br}\right)+\mathrm{H}\right]^{+}\right), 405\left(\left[\mathrm{M}\left({ }^{81} \mathrm{Br}\right)+\mathrm{H}\right]^{+}\right)$. Anal. $\mathrm{C}_{20} \mathrm{H}_{23} \mathrm{BrO}_{2} \mathrm{Si}$ (402.07): found $\mathrm{C} 59.38$, H 5.61 requires $\mathrm{C}$ 59.55, H 5.75.

3-Bromo-2-(2,4-dimethoxyphenyl)benzofuran (8k) (310 mg; 93\%). Yellow solid. Mp 59-61 ${ }^{\circ} \mathrm{C} . \mathrm{R}_{\mathrm{f}}=0.26$ (Cyclohexane/ EtOAc: 9/1). $\mathrm{NMR}^{1} \mathrm{H}\left(\mathrm{CDCl}_{3}, 400 \mathrm{MHz}\right): \delta 3.87\left(\mathrm{~s}, 3 \mathrm{H}, \mathrm{OCH}_{3}\right)$, $3.88\left(\mathrm{~s}, 3 \mathrm{H}, \mathrm{OCH}_{3}\right), 5.61\left(\mathrm{~m}, 2 \mathrm{H}, \mathrm{H}_{\text {arom }}\right), 7.33\left(\mathrm{~m}, 1 \mathrm{H}, \mathrm{H}_{\text {arom }}\right)$, $7.34\left(\mathrm{~m}, 1 \mathrm{H}, \mathrm{H}_{\text {arom }}\right), 7.51\left(\mathrm{~m}, 1 \mathrm{H}, \mathrm{H}_{\text {arom }}\right), 7.53\left(\mathrm{~m}, 1 \mathrm{H}, \mathrm{H}_{\text {arom }}\right)$, $7.57\left(\mathrm{~m}, 1 \mathrm{H}, \mathrm{H}_{\text {arom }}\right)$. NMR ${ }^{13} \mathrm{C}\left(\mathrm{CDCl}_{3}, 100 \mathrm{MHz}\right): \delta 55.6$ $\left(\mathrm{OCH}_{3}\right), 55.8\left(\mathrm{OCH}_{3}\right), 96.2,99.2,104.9,111.2,111.5,119.7$, $123.2,125.0,129.1,132.6,150.8,153.8,159.1,162.6$. IR $\left(v \mathrm{~cm}^{-}\right.$ $\left.{ }^{1}\right): 2938,1616,1499,1449,1209,1161,1033,986,745 . \mathrm{MS}$ $(\mathrm{APCI}+) \mathrm{m} / z$ 254.0 $\left([\mathrm{M}-\mathrm{Br}+\mathrm{H}]^{+}\right)$. Anal. $\mathrm{C}_{16} \mathrm{H}_{13} \mathrm{BrO}_{3}(333.18)$ : found $\mathrm{C}$ 57.55, H 3.66 requires $\mathrm{C}$ 57.68, H 3.93.

(E)-4-(2-(4-Methoxystyryl)benzofuran-3-yl) but-3-yn-1-ol (9) (254 mg; 80\%). A solution of $\mathrm{PdCl}_{2}\left(\mathrm{PPh}_{3}\right)_{2}$ (5 mol\%), CuI (10 mol \%) 2 b (329 mg, $1 \mathrm{mmol})$ and butyn-4-ol (112 mg, 1.6 $\mathrm{mmol})$ in TEA $(10 \mathrm{~mL})$ was stirred at $50{ }^{\circ} \mathrm{C}$ for $12 \mathrm{~h}$. The stirred reaction next treated with saturated $\mathrm{NH}_{4} \mathrm{Cl}$ solution $(25 \mathrm{~mL})$. The aqueous layer was extracted with diethylether $(3 \times 20 \mathrm{~mL})$, the combined organic layers were washed successively with aqueous $\mathrm{HCl}(0.2 \mathrm{M}, 15 \mathrm{~mL}), \mathrm{NaHCO}_{3}(10 \mathrm{~mL})$ and $\mathrm{H}_{2} \mathrm{O}(2 \mathrm{x}$ $25 \mathrm{~mL}$ ), dried over $\mathrm{MgSO}_{4}$ and concentrated under vacuum. Brown oil. $\mathrm{R}_{\mathrm{f}}=0.50$ (Cyclohexane/ EtOAc: 5/5). NMR ${ }^{1} \mathrm{H}$ $\left(\mathrm{CDCl}_{3}, 300 \mathrm{MHz}\right): \delta 2.86\left(\mathrm{t}, J=6.3 \mathrm{~Hz}, 2 \mathrm{H}, \mathrm{CH}_{2}-\mathrm{C}\right), 3.84(\mathrm{~s}$, $3 \mathrm{H}, \mathrm{OMe}), 3.92\left(\mathrm{t}, J=6.3 \mathrm{~Hz}, 2 \mathrm{H}, \mathrm{CH}_{2} \mathrm{O}\right), 6.93(\mathrm{dd}, J=9.0 \mathrm{~Hz}$, $\left.J=2.4 \mathrm{~Hz}, 2 \mathrm{H}, \mathrm{H}_{\text {arom }}\right), 7.06\left(\mathrm{~d}, J=16.2 \mathrm{~Hz}, 1 \mathrm{H}, \mathrm{H}_{\text {vinyl }}\right), 7.63-$ $7.20\left(\mathrm{~m}, 7 \mathrm{H}, \mathrm{H}_{\text {arom+vinyl }}\right)$. NMR ${ }^{13} \mathrm{C}\left(\mathrm{CDCl}_{3}, 100 \mathrm{MHz}\right): \delta 24.5$ $\left(\mathrm{CH}_{2}\right), 55.5\left(\mathrm{CH}_{3}\right), 61.5\left(\mathrm{CH}_{2}\right), 73.1(\mathrm{C}), 94.1(\mathrm{C}), 112.7(\mathrm{CH})$, $114.4(\mathrm{CH}), 120.1(2 \mathrm{CH}), 123.3(\mathrm{CH}), 125.3(\mathrm{CH}), 128.5(\mathrm{CH})$, $129.6(2 \mathrm{CH}), 131.3(\mathrm{CH}), 153.9(\mathrm{C}), 157.8(\mathrm{C}), 160.2(\mathrm{C})$. IR ( $v$ $\left.\mathrm{cm}^{-1}\right): 3448,2935,1734,1602,1577,1508,1452,1423,1373$, 1245, 1034, 959, 932, 746. Anal. $\mathrm{C}_{21} \mathrm{H}_{18} \mathrm{O}_{3}$ (318.37): found $\mathrm{C}$ 79.00, H 5.36 requires C 79.22, H 5.70.

(E)-2-(4-Methoxystyryl)-3-(3,4,5-trimethoxy-phenyl)benzo furan (10) (374 mg; 90\%). To a solution of $\mathbf{2 b}(1 \mathrm{mmol})$ in toluene $(4 \mathrm{~mL})$ and $\mathrm{EtOH}(2 \mathrm{~mL})$, were successively added 3,4,5-trimethoxyphenyl boronic acid (254 mg, $1.2 \mathrm{mmol}$ ), $\mathrm{K}_{2} \mathrm{CO}_{3}(2 \mathrm{mmol})$ and $\mathrm{Pd}\left(\mathrm{PPh}_{3}\right)_{4}(0.05 \mathrm{mmol})$. The reaction was heated at $100{ }^{\circ} \mathrm{C}$, under vigorous stirring and monitored by TLC until complete disappearance of starting material. The solvent was evaporated in vacuo and $10 \mathrm{~mL}$ of water were added. After 
extraction with $\mathrm{CH}_{2} \mathrm{Cl}_{2}(3 \times 10 \mathrm{~mL})$, the combined organic layers were dried over $\mathrm{MgSO}_{4}$ and the solvent was removed under reduced pressure. The crude material was purified by column chromatography to afford 10. Yellow oil. $\mathrm{R}_{\mathrm{f}}=0.25$ (Cyclohexane/ EtOAc: 8/2). NMR ${ }^{1} \mathrm{H} \quad\left(\mathrm{CDCl}_{3}, \quad 300\right.$ $\mathrm{MHz}): \delta 3.72\left(\mathrm{~s}, 3 \mathrm{H}, \mathrm{OCH}_{3}\right), 3.82\left(\mathrm{~s}, 6 \mathrm{H}, \mathrm{OCH}_{3}\right), 3.90(\mathrm{~s}, 3 \mathrm{H}$, $\left.\mathrm{OCH}_{3}\right), 6.71$ (s, 2H, $\left.\mathrm{H}_{\text {arom }}\right), 6.80$ (d, J=7.8 Hz, 2H, $\left.\mathrm{H}_{\text {arom }}\right), 6.98$ $\left(\mathrm{d}, J=16.2 \mathrm{~Hz}, 1 \mathrm{H}, \mathrm{H}_{\text {vinyl }}\right), 7.15-7.7 .30\left(\mathrm{~m}, 2 \mathrm{H}, \mathrm{H}_{\text {arom }}\right), 7.35$ (d, $\left.J=16.2 \mathrm{~Hz}, 1 \mathrm{H}, \mathrm{H}_{\text {vinyl }}\right), 7.42\left(\mathrm{~d}, J=7.8 \mathrm{~Hz}, 2 \mathrm{H}, \mathrm{H}_{\text {arom }}\right), 7.36-$ $7.60\left(\mathrm{~m}, 2 \mathrm{H}, \mathrm{H}_{\text {arom }}\right) . \mathrm{NMR}{ }^{13} \mathrm{C}\left(\mathrm{CDCl}_{3}, 100 \mathrm{MHz}\right): \delta 55.3\left(\mathrm{CH}_{3}\right)$, $56.3\left(2 \mathrm{CH}_{3}\right), 61.0\left(\mathrm{CH}_{3}\right), 106.6(2 \mathrm{CH}), 110.9(\mathrm{CH}), 112.9(\mathrm{CH})$, $114.3(2 \mathrm{CH}), 118.8(\mathrm{C}), 119.7(\mathrm{CH}), 123.0(\mathrm{CH}), 124.9(\mathrm{CH})$, $127.9(\mathrm{C}), 128.0(2 \mathrm{CH}), 129.1(\mathrm{C}), 129.5(\mathrm{C}), 130.5(\mathrm{CH}), 137.5$ (C), $151.1(\mathrm{C}), 153.6(\mathrm{C}), 154.2(\mathrm{C}), 159.8(\mathrm{C}) . \mathrm{IR}\left(v \mathrm{~cm}^{-1}\right)$ : 2932, 2249, 2068, 1602, 1580, 1509, 1452, 1414, 1389, 1307, 1243, 1173, 1125, 1032, 1005, 961, 907, 843, 819. Anal. $\mathrm{C}_{26} \mathrm{H}_{24} \mathrm{O}_{5}$ (416.47): found $\mathrm{C} 74.89, \mathrm{H} 5.66$ requires $\mathrm{C} 74.98, \mathrm{H}$ 5.81 .

\section{Acknowledgment}

The Centre National de la Recherche Scientifique (CNRS) is gratefully acknowledged for financial support of this research and Ministère de la Recherche et de l'Enseignement Supérieur (MRES) for a doctoral fellowship to M. J. Thanks also to the Association de Recherche sur le Cancer (ARC) for a post-doctoral grant to. A. T. and for financial support. Thanks also to E. Morvan for her help in the NMR experiments.

\section{Notes and references}

Received: ((will be filled in by the editorial staff)) Published online: ((will be filled in by the editorial staff))

[1] a) F. Liron, P. Le Garrec, M. Alami Synlett 1999, 246-248; b) M. Alami, F. Liron, M. Gervais, J.-F. Peyrat, J.-D. Brion Angew. Chem. Int. Ed. 2002, 41, 1578-1580; c) A. Hamze, O. Provot, M. Alami, J.-D. Brion Org. Lett. 2005, 7, 5625-5628; d) A. Hamze, O. Provot, J.-D. Brion, M. Alami Synthesis 2007, 2025-2036; e) A. Giraud, O. Provot, A. Hamze, J.-D. Brion, M. Alami Tetrahedron Lett. 2008, 49, 1107-1110; f) A. Hamze, O. Provot, J.-D. Brion, M. Alami Tetrahedron Lett. 2008, 49, 2429-2431; g) A. Hamze, O. Provot, J.-D. Brion, M. Alami J. Organomet. Chem. 2008, 693, 2789-2797; h) C. Mousset, O. Provot, A. Hamze, J. Bignon, J.-D. Brion, M. Alami Tetrahedron 2008, 64, 4287-4294; i) C. Mousset, A. Giraud, O. Provot, A. Hamze, J. Bignon, J.-M. Liu, S. Thoret, J. Dubois, J.-D. Brion, M. Alami Bioorg. Med. Chem. Lett. 2008, 18, 3266-3271.

[2] M. Jacubert, O. Provot, J.-F. Peyrat, A. Hamze, J.-D. Brion, M. Alami Tetrahedron 2010, 66, 3775-3787.

[3] a) N. Olivi, E. Thomas, J.-F. Peyrat, M. Alami, J.-D. Brion Synlett 2004, 2175-2179; b) G. Le Bras, O. Provot, J.-F. Peyrat, M. Alami, J.-D. Brion Tetrahedron Lett. 2006, 47, 5497-5501.

[4] a) G. Le Bras, A. Hamze, S. Messaoudi, O. Provot, P.-B. Le Calvez, J.-D. Brion, M. Alami Synthesis 2008, 1607-1611; b) M. Jacubert, A. Hamze, O. Provot, J.-F. Peyrat, J.-D. Brion, M. Alami Tetrahedron Lett. 2009, 50, 3588-3592.

[5] Benzofuran derivatives have been also prepared from the cyclization of alkynyl phenols see: a) A. S. K. Hashmi, T. M. Frost, J. W. bats Org. Lett. 2001, 3, 3769-3771. b) A. S. K. Hashmi, E. Enns, T. M. Frost, S. Schäfer, W. Frey, F. Rominger Synthesis 2008, 2707-2718. c) W. M. Dai, K. W. Lai Tetrahedron Lett. 2002, 43, 9377-9380.
[6] a) A. R. Katritzsky Comprehensive Heterocyclic chemistry; Pergamon Press: Oxford, 1984, Vol 4, Part 3, p 658; b) A. Williams Furans, Synthesis and Applications; Noyes Data Corporation: Park ridge, NJ, 1973; pp 1-303; c) X.-L. Hou, Z. Yang, H. N. C. Wong Furans and Benzofurans, In Progress in Heterocyclic Chemistry, Vol. 14; G. W. Gribble, T. L. Gilchrist, Eds.; Pergamon: Oxford, 2002, 193-179; d) Z. Qin, I. Kastrati, R. E. P. Chandrasena, H. Liu, P. Yao, P. A. Petukhov, J. L. Bolton, G. R. J. Thatcher J. Med. Chem. 2007, 50, 2682-2692; e) E. P. Santín, H. Khanwalkar, J. Voegel, P. Collette, P. Mauvais, H. Gronemeyer, A. R. de Lara ChemMedChem 2009, 4, 780-791; f) C.-L. Kao, J.-W. Chern, J. Org. Chem. 2002, 67, 6772-6787; g) G. F. Filzen, L. Bratton, X.-M. Cheng, N. Erasga, A. Geyer, C. Lee, G. Lu, J. Pulaski, R. J. Sorenson, P. C. Unangst, B. K. Trivedi, X. Xu Bioorg. Med. Chem. Lett. 2007, 17, 3630-3635.

[7] a) K. G. Pinney, A. D. Bounds, K. M. Dingeman, V. P. Mocharla, G. R. Pettit, R. Bai, E. Hamel Bioorg. Med. Chem. Lett.1999, 9, 1081-1086; b) K. G. Pinney, G. R. Pettit, V. P. Mocharla, P. M. Del, A. Shirali PCT Int. Appl. WO 9839323; Chem. Abstr. 1998, 129, 245037c; c) Z. Chen, V. Mocharla, J. M. Farmer, G. R. Pettit, E. Hamel, K. G. Pinney J. Org. Chem. 2000, 65, 8811-8815.

[8] a) S. Messaoudi, B. Tréguier, A. Hamze, O. Provot, J.-F. Peyrat, J. R. Rodrigo De Losada, J.-M. Liu, J. Bignon, J. WdzieczakBakala, S. Thoret, J. Dubois, J.-D. Brion, M. Alami J. Med. Chem. 2009, 52, 4538-4542; b) M. Alami, J.-D. Brion, O. Provot, J.-F. Peyrat, S. Messaoudi, A. Hamze, A. Giraud, J. Bignon, J. Bakala, J.-M. Liu WO 122620 A1, 2008; c) O. Provot, A. Giraud, J.-F. Peyrat, M. Alami, J.-D. Brion Tetrahedron Lett. 2005, 46, 8547-8550; d) A. Hamze, A. Giraud, S. Messaoudi, O. Provot, J.F. Peyrat, J. Bignon, J.-M. Liu, J. Wdzieczak-Bakala, S. Thoret, J. Dubois, J.-D. Brion, M. Alami ChemMedChem. 2009, 4, 1912 1924; e) G. Le Bras, C. Radanyi, J.-F. Peyrat, J.-D. Brion, M Alami, V. Marsaud, B. Stella, J.-M. Renoir J. Med. Chem. 2007, 50, 6189-6200.

[9] a) F. Toda, M. Nakagawa Bull. Chem. Soc. Jpn 1960, 33, 12871291; b) Y. Liang, S. Tang, X.-D. Zhang, L.-Q. Mao, Y.-X. Xie J.-H. Li Org. Lett. 2006, 8, 3017-3020. c) J. P. Weyrauch, A. S. K. Hashmi, A. Schuster, T. Hengst, S. Schetter, A. Littmann, M Rudolph, M. Hamzic, J. Visus, F. Rominger, W. Frey, J. W. Bats Chem. Eur. J. 2010, 16, 956-963. d) L.-Y. Yang, C.-F. Chang, Y.-C. Huang, Y.-J. Lee, C.-C. Hu, T.-H. Tseng Synthesis 2009, 1175-1179. e) H. Zhou, J.-J. Niu, J.-W. Xu, S.-J. Hu Synthetic Commun. 2009, 39, 716-732. f) Y. Liang, S. Tang, X.-D. Zhang, Y.-Q. Mao, Y.-X. Xie, J.-H. Li Org. Lett. 2006, 8, 3017-3020. g) A. Arcadi, S. Cacchi, G. Fabrizi, F. Marinelli, L. Moro Synlett 1999, 1432-1434. h) P. K. Mohakhud, M. R. Parthasarathy Indian J. Chem. B 1995, 34B, 713-717. i) D. R. Buckle, C. J. M. Rockell J. Chem. Soc. Perkin Trans. 1 1985, 2443-2446.

[10] a) S. Mehta, J. P. Waldo, R. C. Larock J. Org. Chem. 2009, 74, 1141-1147; b) T. Okitsu, D. Nakazawa, R. Taniguchi, A. Wada Org. Lett. 2008, 10, 4967. c) F. Manarin, J. A. Roehrs, R. M Gay, R. Brandao, P. H. Menezes, C. W. Nogueira, G. Zeni J. Org. Chem. 2009, 74, 2153-2162. d) H. B. Bang, S. Y. Han, D. H Choi, J. W. Hwang, J.-G. Jun Arkivok 2008, 2, 112-125. e) C.-H. Cho, B. Neuenswander, G. H. Lushingto,; R. C. Larock J. Comb. Chem. 2008, 10, 941-947. f) H. B. Bang, S. Y. Han, D. H. Choi, D. M. Yang, J. W. Hwang, H. S. Lee, J.-G. Jun Synthetic Commun. 2009, 39, 506-515. g) T. Okitsu, D. Nakazawa, R. Taniguchi, A. Wada Org. Lett. 2008, 10, 4967-4970. h) N. Ahmed, C. Dubuc, J. Rousseau, F. Bénard, E. van Lier Bioorg. Med. Chem. Lett. 2007, 17, 3212-3216. i) D. Yue, T. Yao, R. C. Larock J. Org. Chem. 2005, 70, 9985-9989. j) F. Colobert, A.-S. Castanet, O. Abillard Eur. J. Org. Chem. 2005, 3334-3341. k) T. Yao, D. Yue, R. C. Larock J. Comb. Chem. 2005, 7, 809-812. 1) C.-L. Kao, J.-W. Chern J. Org. Chem. 2002, 67, 6772-6787.

[11] a) B. L. Flynn, P. Verdier-Pinard, E. Hamel Org. Lett. 2001, 5, 651; b) R. C. Larock, D. Yue Tetrahedron Lett. 2001, 42, 60116013; c) D. Yue, R. C. Larock J. Org. Chem. 2002, 67, 19051909; d) W.-D. Lu, M.-J. Wu Tetrahedron 2007, 63, 356-362.

[12] a) A. Bekaert, O. Barberan, E. B. Kaloun, A. Danan, J.-D. Brion, P. Lemoine, B. Viossat Z. Kristallogr. NCS 2001, 216, 1-2; b) J.F. Berrien, O. Provot, D. Joseph, A. Bekaert J. Chem. Educ. 2004, 
81, 1348-1349; c) A. Bekaert, O. Provot, O. Rasolojaona, M. Alami, J.-D. Brion Tetrahedron Lett. 2005, 46, 4187-4191.

[13] a) A. Tikad, A. Hamze, O. Provot, J.-D. Brion, M. Alami Eur. J. Org. Chem. 2010, 725-731; b) P. Ramiandrasoa, B. Bréhon, A. Thivet, M. Alami, G. Cahiez, Tetrahedron Lett. 1997, 38, $2447-$ 2450; c) M. Seck, X. Franck, R. Hocquemiller, B. Figadère, J.-F. Peyrat, O. Provot, J.-D. Brion, M. Alami, Tetrahedron Lett. 2004, 45, 1881-1884; d) M. Dos Santos, X. Franck, R. Hocquemiller, B. Figadère, J.-F. Peyrat, O. Provot, J.-D. Brion, M. Alami, Synlett 2004, 2697-2700; e) A. Hamze, O. Provot, J.-D. Brion, M. Alami, J. Org. Chem. 2007, 72, 3868-3874; f) M. Alami, P. Ramiandrasoa, G. Cahiez, Synlett 1998, 325-327.

[14] M. Alami, F. Ferri Tetrahedron Lett. 1996, 37, 2763-2766.

[15] Y. Dienes, M. Eggenstein, T. Kárpáti, T. Sutherland, L. Nyulászi, T. Baumgartner Chem. Eur. J. 2008, 14, 9878-9889.

[16] G. Balaji, S. Valiyaveettil Org. Lett. 2009, 11, 3358-3361.

[17] J. Ohshita, K.-H. Lee, K. Kimura, A. Kunai, Organometallics 2004, 23, 5622-5625.

[18] M. Shimizu, I. Nagao, Y. Tomioka, T. Hiyama Angew. Chem. Int. Ed. 2008, 47, 8096-8099.

[19] a) M. Alami, B. Crousse; G. Linstrumelle, Tetrahedron Lett. 1994, 35, 3543-3546; b) F. Ferri, M. Alami Tetrahedron Lett. 1996, 37, 7971-7974.

[20] a) H. Shirani, T. Janosik J. Org. Chem. 2007, 72, 8984-8986; b) E. Wincent, H. Shirani, J. Bergman, U. Rannung, T. Janosik Bioorg. Med. Chem. 2009, 17, 1648-1653.

[21] H. Shirani, T. Janosik Organometallics 2008, 27, 3960-3963.

[22] a) K. Sonogashira, Y. Tohda, N. Hagihara Tetrahedron Lett. 1975, 4467-4470; b) M. Alami, F. Ferri, G. Linstrumelle Tetrahedron Lett. 1993, 34, 6403-6406. (c) M. Alami, F. Ferri, Y. Gaslain Tetrahedron Lett. 1996, 37, 57-60.

[23] J. Kaffy, R. Pontikis, J.-C. Florent, C. Monneret Org. Biomol. Chem. 2005, 3, 2657-2660.

[24] R. D. Coulson Inorg. Synth. 1972, 13, 121-124.

[25] S. Mehta, R. C. Larock J. Org. Chem. 2010, 75, 1652-1658.

[26] F. Toda, M. Nakagawa Bull Chem. Soc Jpn. 1960, 33, 1287-1291.

[27] W. Schroth, E. Hintzsche, H. Jordan, T. Jende, R. Spitzner, I. Thondorf Tetrahedron 1997, 53, 7509-7528.

[28] T. Bach, M. Bartels Synlett 2001, 1284-1286.

[29] W.-D. Lu, M.-J. Wu Tetrahedron 2006, 63, 356-362. 